\title{
Optimal Lockdown in a Commuting Network*
}

\author{
Pablo D. Fajgelbaum ${ }^{1}$, Amit Khandelwal ${ }^{2}$, Wookun $\mathrm{Kim}^{3}$, Cristiano Mantovani ${ }^{4}$, and \\ Edouard Schaal ${ }^{5}$ \\ ${ }^{1}$ Princeton and NBER \\ ${ }^{2}$ Columbia GSB and NBER \\ ${ }^{3}$ Southern Methodist University \\ ${ }^{4}$ Universitat Pompeu Fabra \\ ${ }^{5}$ CREI, ICREA, UPF, BGSE and CEPR
}

November 13, 2020

\begin{abstract}
We study optimal dynamic lockdowns against Covid-19 within a commuting network. Our framework integrates canonical spatial epidemiology and trade models, and is applied to cities with varying initial viral spread: Seoul, Daegu and NYC-Metro. Spatial lockdowns achieve substantially smaller income losses than uniform lockdowns. In NYM and Daegu - with large initial shocks - the optimal lockdown restricts inflows to central districts before gradual relaxation, while in Seoul it imposes low temporal but large spatial variation. Actual commuting reductions were too weak in central locations in Daegu and NYM, and too strong across Seoul.
\end{abstract}

JEL Classification: R38, R4, C6

\footnotetext{
*E-mail: pfajgelb@princeton.edu, ak2796@columbia.edu, wookunkim@smu.edu, eschaal@crei.cat, cristiano.mantovani@upf.edu. We thank Andy Atkeson for his comments, as well as the editor and two anonymous referees. Schaal acknowledges financial support from the Spanish Ministry of Economy and Competitiveness, through the Severo Ochoa Programme for Centres of Excellence in R\&D (SEV-2015-0563) and the European Research Council Starting Grant 804095. We thank SafeGraph for making their data freely available. We thank Hyungmo Choi for providing excellent research assistance.
} 


\section{Introduction}

Commuting networks are the backbone of cities, allowing interactions that are vital for economic growth. On a typical day, Manhattan receives as many commuters as its residents-about 1.6 million people. Two months after the onset of Covid-19, NYC metro commute flows were $49 \%$ below prepandemic levels. Weighing the economic costs against the benefits of stopping Covid-19, was this reduction too large or not large enough? To fight a highly infectious disease without a vaccine, public authorities must decide if and how to curtail movements across locations connected via commuting and trade. ${ }^{1}$ How should lockdown policies be set across locations and time?

In this paper we establish an efficient benchmark against which to measure the losses from uncoordinated or spatially uniform lockdown efforts. We study optimal dynamic lockdowns to fight pandemics in a commuting network using a framework that integrates standard spatial epidemiology and trade models. ${ }^{2}$ In the model, a disease spreads through interactions of commuters at the workplace. Lockdown policies directly reduce the real income of workers who stay at home and increase shopping costs, and indirectly impact other locations through shifts in expenditures. ${ }^{3}$ In reality, policies that close specific businesses preclude commutes along particular routes. Our planning problem determines the fraction of each origin-destination commuting flow allowed to operate at each point in time to minimize the economic costs and the loss of lives. We also implement optimal lockdowns by origin or destination that resemble less flexible closures (e.g., lockdowns by neighborhood).

We apply the model using real-time commuting data across districts in two South Korean cities, Seoul and Daegu, and cellphone mobility data across counties in the NYC Metro area (NYM). We compare optimal pandemic-fighting strategies across intensities of the initial virus shock and contrast them with the observed commuting responses. We analyze Korean cities because Korea has tested for Covid-19 at greater intensities than most countries, making the timeline of their case data more reliable. ${ }^{4}$ Seoul is the largest city in Korea and experienced a relatively small caseload, while Daegu (Korea's fourth-largest city) experienced the country's largest shock. We study NYM because of its economic importance and rapid spread.

We compute the optimal lockdown given the Covid-19 spread when lockdown policies were announced. The model matches pre-pandemic commuting flows and wages across locations (Korean districts and NYM counties). We estimate the transmission rate using data on the spatial distribu-

\footnotetext{
${ }^{1}$ Lockdowns were announced fairly uniformly across bordering U.S. states, with a mean difference of 4 days, although there has been variation in county-level policies. For example, New York, New Jersey, and Connecticut imposed almost simultaneous lockdown in March 2020, while Illinois did so more two weeks before Missouri (Raifman et al., 2020).

${ }^{2}$ The spatial SIR model we formulate is closely related to the multi-city epidemic model in Arino and Van den Driessche (2003), in which the disease is transmitted from infected residents of location $i$ to susceptible residents of location $j$ when they meet in location $k$. The trade model follows Anderson and Van Wincoop (2003).

${ }^{3}$ Caliendo et al. (2018) and Monte et al. (2018) study diffusion of local shocks across and within cities in related gravity models.

${ }^{4}$ Korea had performed 0.878 tests per thousand people at the time of its 1000 th patient compared to 0.086 in the U.S. Stock (2020), Manski and Molinari (2020), Korolev (2020), and Atkeson (2020a) discuss challenges arising from infrequent testing.
} 
tion of new cases and commuting flows over time. We use geocoded credit card expenditure data from Seoul to estimate the impact of lockdown on the travel costs of shopping.

Our first results show that in NYM and Daegu - where the virus initially spread very quickly locations with high virus-diffusion potential are subject to a strict initial lockdown, eliminating up to $70 \%$ of pre-pandemic inflows, which is partially relaxed over 3 to 6 months. In NYM, many locations are locked down early, but only the top-3 central locations (Manhattan, Brooklyn, and Bronx) remain closed for a long time in expectation that a vaccine arrives. In contrast, in Seoulwhere the initial spread of Covid-19 was much smaller - the planner initially locks down only a few locations of relatively high centrality. As the virus spreads, the lockdown intensifies and retains considerable spatial variation.

Our main result reveals large benefits from spatial targeting. Specifically, we find substantially lower real-income losses from spatial targeting compared to an optimal but spatially uniform lockdown. Given the actual case count by April 30, spatial targeting would have led to $20 \%, 32 \%$, and $55 \%$ lower economic costs in Daegu, Seoul, and NYM, respectively, than the optimal uniform lockdown. We find that optimal lockdowns by destination of commuting flows are almost as efficient as the fully flexible benchmark, suggesting that spatially targeted business lockdowns may be enough to reap the benefits of spatial targeting.

Finally, we compare the optimal benchmark with the observed commuting reductions resulting from government action and commuters' precautionary behavior. On average across locations, commuting declines reached troughs of $79 \%, 36 \%$, and $82 \%$ below pre-pandemic levels in Daegu, Seoul, and NYM before modestly reverting upward. In NYM and Daegu, these city-level declines are not far from the optimal benchmark. However, the most central (peripheral) locations exhibited a weaker (stronger) reduction in commuting than what would have been optimal. Across Seoul, the actual commuting reductions were too strong compared to the optimal. As a result, across all three cities, the real income losses could have been much smaller through optimal spatial targeting.

Studies of optimal epidemic control in economic models include Goldman and Lightwood (2002) and Rowthorn and Toxvaerd (2012) and, in the context of Covid-19, Atkeson (2020b), Alvarez et al. (2020), Jones et al. (2020), Piguillem and Shi (2020), Rowthorn (2020), and Rowthorn and Toxvaerd (2020), among others. Acemoglu et al. (2020), Baqaee et al. (2020), and Glover et al. (2020) among others study lockdown with heterogeneous agents.

Adda (2016) demonstrates that diseases spread through transportation networks exploiting variation from public-transport strikes in France, and Viboud et al. (2006) show that work-related flows correlate with influenza's regional spread in the United States. For Covid-19, Tian et al. (2020) argue that the Wuhan lockdown and suspending public transport delayed the spread across China, Fang et al. (2020) show that the lockdown reduced infection rates using real-time movement data, Kissler et al. (2020) show that commuting correlates with cases within New York, and Hsiang et al. (2020) and Flaxman et al. (2020) show that interventions like lockdown reduced the spread. Spatial SIR models were first used to study influenza and measles (Rvachev and Longini Jr, 1985; Bolker and Grenfell, 1995). Germann et al. (2006), Eubank et al. (2004) and Drakopolous 
and Zheng (2017) study targeted policies in spatial or network models, and Rowthorn et al. (2009) analyze their theoretical properties. For Covid-19, Chinazzi et al. (2020), Birge et al. (2020) and Giannone et al. (2020) simulate location specific policies, Argente et al. (2020) consider case information disclosure, and Antràs et al. (2020) study pandemics in a trade model with human interactions.

Our contribution is three-fold. First, we implement optimal lockdown over both time and space in a commuting network. Second, to evaluate the diffusion of economic costs through changes in spending we integrate a general-equilibrium trade framework. Third, we use real-time commuting and expenditure data to estimate and compare the actual commuting responses over space with optimal lockdowns.

\section{Model}

We use a standard spatial epidemiology model similar to Arino and Van den Driessche (2003). The general equilibrium corresponds to a standard quantitative gravity trade model (e.g., Anderson and Van Wincoop 2003 and Eaton and Kortum 2002).

\subsection{Spatial Diffusion}

The economy consists of $J$ locations in continuous time. Before the pandemic, in each location $i$ there are $N_{0}(i)$ residents, of which a fraction $\lambda(i, j)$ commutes to $j$. We let $\boldsymbol{\Lambda}$ be the matrix of bilateral commuting flows such that $[\boldsymbol{\Lambda}]_{i j}=\lambda(i, j) .{ }^{5}$

At each time $t$, the surviving residents of location $i$ are either susceptible, exposed, infected, or recovered in quantities $S(i, t), E(i, t), I(i, t)$, and $R(i, t)$, respectively. Susceptible agents become exposed after interacting with infected agents; exposed agents are latent carriers who do not infect others and become infected at rate $\gamma_{I}$. Infected agents die at rate $\gamma_{D}$ or recover (and become immune) at rate $\gamma_{R}$. The spatial distributions are collected in the (column) vectors $\mathbf{S}(t), \mathbf{E}(t)$, $\mathbf{I}(t)$, and $\mathbf{R}(t)$.

The government can control the fraction $\chi(i, j, t)$ of commuting flows from $i$ to $j$ by imposing lockdown measures, providing incentives, or broadcasting information. ${ }^{6}$ Every agent is subject to the policy regardless of infection status. Among those not infected and not physically commuting, a fraction $\delta$ telecommutes. Among the infected, only the asymptomatic fraction $\zeta$ works. The lockdown policies are collected in the $J$ by $J$ matrix $\chi(t)$.

The geographic spread of the disease depends on how much infected and susceptible people interact in space. The infections could happen anywhere at commuting locations, such as in train

\footnotetext{
${ }^{5}$ These initial distributions could be the equilibrium of a spatial model as in Redding and Rossi-Hansberg (2017). Given our timeframe we assume no job switches other than through lockdown.

${ }^{6} \mathrm{An}$ implicit assumption is that policymakers can randomly test to obtain location-specific distributions of each infection status, but cannot observe every individual's status. Such random testing would also mitigate potential errors in testing (e.g., Manski and Molinari 2020).
} 
stations, workplaces, or restaurants. In this benchmark the virus diffuses only through commuting. In alternative specifications we also allow the virus to spread through shopping.

Let $\tilde{S}(j, t) \equiv \sum_{i^{\prime}} \chi\left(i^{\prime}, j, t\right) \lambda\left(i^{\prime}, j\right) S\left(i^{\prime}, t\right)$ be the number of susceptible agents (from any origin) exposed in $j$, and $\tilde{I}(j, t) \equiv \zeta \sum_{i^{\prime}} \chi\left(i^{\prime}, j, t\right) \lambda\left(i^{\prime}, j\right) I\left(i^{\prime}, t\right)$ be the number of infected asymptomatic agents (from any origin) spreading the disease in $j$. In matrix form, $\tilde{\mathbf{S}}(t) \equiv \mathbf{H}_{S}(t) \mathbf{S}(t)$ and $\tilde{\mathbf{I}}(t) \equiv \mathbf{H}_{I}(t) \mathbf{I}(t)$, where $\mathbf{H}_{S}(t)$ and $\mathbf{H}_{I}(t)$ are spatial incidence matrices that depend on prepandemic commuting flows and current lockdown policies:

$$
\mathbf{H}_{u}(\chi(t))=\zeta_{u}(\boldsymbol{\Lambda} \cdot \chi(t))^{\prime}
$$

where $\zeta_{I}=\zeta, \zeta_{S}=1$, and is the element-wise product. The $(j i)$ element of $\mathbf{H}_{S}(t)$ is the exposure of the $S(i, t)$ susceptible residents of location $j$ to the $\tilde{I}(j, t)$ infected commuters to $j$. Similarly, the $(j i)$ element of $\mathbf{H}_{I}(t)$ is the exposure of the $I(i, t)$ infected residents of location $j$ to the $\tilde{S}(j, t)$ susceptible commuters to $j$.

The flow of new infections in $j$ is

$$
M_{j}(\tilde{I}(j, t), \tilde{S}(j, t))
$$

where $M_{j}(\cdot)$ represents the matching process between infected and susceptible individuals in $j$. The infections taking place in $j$ are carried back by susceptible and infected agents to their residence. Of these infections, a fraction $H_{S}(j, i, t) S(i, t) / \tilde{S}(j, t)$ corresponds to residents of $i$. Therefore, the flow of the new infections among location $i$ 's residents is:

$$
\dot{S}(i, t)=-\sum_{j} \frac{H_{S}(j, i, t) S(i, t)}{\tilde{S}(j, t)} M_{j}(\tilde{I}(j, t), \tilde{S}(j, t))
$$

\subsection{Real Income}

The economic costs of lockdown enter through the distribution of real income,

$$
U(i, t)=\frac{Y(i, t)}{P(i, t)}
$$

where $P(i, t)$ is the cost of living and $Y(i, t)$ is the nominal income of location- $i$ residents:

$$
Y(i, t)=\sum_{u=S, E, I, R} \sum_{j} N_{u}(i, j, t) w(j, t)
$$

Here, $w(j, t)$ is the wage per efficiency unit in $j$ at time $t$ and $N_{u}(i, j, t)$ is the flow of efficiency units of type- $u$ commuters from $i$ to $j$ :

$$
N_{u}(i, j, t)=\zeta_{u}[\chi(i, j, t)+(1-\chi(i, j, t)) \delta] \lambda(i, j) u(i, t)
$$


for $u=S, E, I, R$, where $\zeta_{I}=\zeta$ is the fraction of asymptomatic infected and $\zeta_{u}=1$ for $u \neq I$. The efficiency units flowing from $i$ to $j$ include those physically commuting, $\chi(i, j, t)$, and those not commuting scaled by the fraction of telecommuters, $(1-\chi(i, j, t)) \delta$.

The jobs at $j$ produce goods or services with productivity $z(j)$. Consumers have a constant elasticity of substitution $\sigma$ across goods from different locations. Residents of $j$ face $\operatorname{costs} \tau(i, j, t) \equiv$ $\tau(i, j, \chi(t))>1$ when shopping in $i$. The lockdown restrictions increase this shopping cost.

In equilibrium, markets clear at all $t$ :

$$
w(i, t) \sum_{u=S, E, I, R} \sum_{j} N_{u}(j, i, t)=\sum_{j} s(i, j, t) Y(j, t) \text { for all } i
$$

i.e., the total income of workers employed in $i$ equals the aggregate expenditures in goods from $i$, where

$$
s(i, j, t) \equiv\left(\frac{p(i, j, t)}{P(j, t)}\right)^{1-\sigma}
$$

is the expenditure share of goods from $i$ in location $j$ given the price $p(i, j, t)=\tau(i, j, t) \frac{w(i, t)}{z(i)}$, and

$$
P(j, t)=\left(\sum_{i} p(i, j, t)^{1-\sigma}\right)^{\frac{1}{1-\sigma}}
$$

is the price index in $j$. In equilibrium, $\{w(j, t), P(j, t)\}$ are such that (7) and (9) hold.

\subsection{Planning Problem}

A social planner chooses the lockdown matrix to maximize the present discounted value of real income net of loss of lives. The aggregate real income of location $j$ depends on the spatial distributions of lockdown and residents by infection status:

$$
U(j, t) \equiv U(j ; \mathbf{S}(t), \mathbf{E}(t), \mathbf{I}(t), \mathbf{R}(t), \boldsymbol{\chi}(t))
$$

A vaccine and a cure become freely available with probability $\nu$ in every time period. If the cure occurs at time $t$, location $j$ generates the real income $\bar{U}(j, t) \equiv U\left(j ; 0,0,0, \mathbf{S}(t)+\mathbf{E}(t)+\mathbf{I}(t)+\mathbf{R}(t), \mathbf{1}_{J \times J}\right)$ forever. The planning problem is: ${ }^{7}$

$$
W=\max _{\chi(t)} \int_{0}^{\infty} e^{-(r+\nu) t} \sum_{j}\left[U(j, t)+\frac{\nu}{r} \bar{U}(j, t)-\omega \gamma_{D} I(j, t)\right] d t
$$

${ }^{7}$ The flow value for the planner's utility from location $j$ is:

$$
W_{0}(j, t)=\left(U(j, t)-\omega \gamma_{D} I(j, t)\right) d t+\nu d t e^{-r d t} \frac{\bar{U}(j, t)}{r}+e^{-r d t}(1-\nu d t) W_{0}(j, t+d t) .
$$

This value includes the payoff $U(j, t)-\omega \gamma_{D} I(j, t)$, the probability $\nu d t$ of transitioning to a vaccine and its value $\frac{\bar{U}(j, t)}{r}$, and the continuation value. Solving for the present discounted value for location $j$ and adding up across locations yields (11). 
subject to

$$
\begin{aligned}
\dot{\mathbf{S}}(t) & =-\mathbf{S}(t) \cdot\left[\mathbf{H}_{S}(\boldsymbol{\chi}(t))^{\prime}(\mathbf{M}(\tilde{\mathbf{S}}(t), \tilde{\mathbf{I}}(t)) \cdot / \tilde{\mathbf{S}}(t))\right] \\
\dot{\mathbf{E}}(t) & =-\dot{\mathbf{S}}(t)-\gamma_{I} \mathbf{E}(t), \\
\dot{\mathbf{I}}(t) & =\gamma_{I} \mathbf{E}(t)-\left(\gamma_{R}+\gamma_{D}\right) \mathbf{I}(t), \\
\dot{\mathbf{R}}(t) & =\gamma_{R} \mathbf{I}(t)
\end{aligned}
$$

where $\mathbf{M}(\cdot)$ is a vector with the new matches, and where $\tilde{\mathbf{S}}(t)=\mathbf{H}_{S}(\boldsymbol{\chi}(t)) \mathbf{S}(t)$ and $\tilde{\mathbf{I}}(t)=$ $\mathbf{H}_{I}(\boldsymbol{\chi}(t)) \mathbf{I}(t)$ are the susceptible and infected agents. ${ }^{8}$ The matrix $\boldsymbol{\chi}(t)$ impacts aggregate real income and the spatial incidence matrix through (1) and (10).

Following standard SEIR models, we impose a multiplicative matching function,

$$
M_{j}(\tilde{I}, \tilde{S})=\beta_{j} \tilde{I} \tilde{S}
$$

where $\beta_{j}$ is a location-specific diffusion rate. For intuition about the planner's incentives, fix the wage distribution and consider a case where lockdowns do not impact shopping costs. Then the interior solution of the FOC with respect to $\chi(i, j, t)$ is:

$$
\begin{aligned}
(1-\delta) w(j) & =\Delta(i, t) \frac{S(i, t)}{N(i, t)} \beta_{j} \sum_{i^{\prime}} \zeta I\left(i^{\prime}, t\right) \lambda\left(i^{\prime}, j\right) \chi\left(i^{\prime}, j, t\right) \\
& +\frac{\zeta I(i, t)}{N(i, t)} \beta_{j} \sum_{k} \Delta(k, t) S(k, t) \lambda(k, j) \chi(k, j, t),
\end{aligned}
$$

where $N(i, t)$ is the surviving population of $i$ at time $t$ and $\Delta(i, t) \equiv \mu_{S}(i, t)-\mu_{E}(i, t)$, the difference between the co-states of (12) and (13), represents the value of avoiding exposure.

On the left, the economic costs of lockdown equal the wage losses of workers who telecommute. On the right, the first line captures the benefits of deterring susceptible agents in $i$ from commuting and therefore be exposed to infected agents at destination. Similarly, the second line captures that the lockdown deters infected agents from $i$ from transmitting the disease in $j$. The condition highlights the critical tradeoff for the planner: central locations are likely to have higher wages and be hubs for transmission. So, whether they should endure stricter lockdowns depends on the relative importance of economic and health costs.

\section{Data and Parametrization}

The parametrization uses case data and real-time commuting flows to estimate the virus transmission rate. The optimization is implemented starting at the lockdown announcement date using pre-pandemic data on commuting flows, wages, population, and spending. Our units of analysis are the 25 districts in Seoul and 8 districts in Daegu. We define NYM to be 20 counties: 5 NYC

\footnotetext{
${ }^{8}$ The notation $\cdot /$ stands for element-wise ratio.
} 
boroughs, 5 counties in NY (Putnam, Rockland, Westchester, Nassau, Suffolk), 8 counties in New Jersey (Bergen, Essex, Hudson, Middlesex, Morris, Passaic, Somerset, Union), and 2 counties in Connecticut (Fairfield, New Haven).

\subsection{Data}

\section{Covid-19 Data}

The Seoul Metropolitan government released patient-level case data. We filed an Official Information Disclosure Act request to obtain patient-level data from the Daegu Metropolitan government. We build a daily panel dataset with total confirmed cases in the Seoul and Daegu districts. County-level NYM cases come from Johns Hopkins University and the NY State Department of Health. The Korean data allow us to exclude cases arriving from overseas travels that were stopped at the border.

There are limitations to using data on Covid-19 infections due to bias of the tested population and sensitivity of the tests. These limitations are more severe at the onset of the pandemic when testing intensity was low. Our estimation of the virus transmission rate uses data from the later periods when reporting and testing improved. Additionally, in Korea, testing intensity has been high since the onset.

\section{Daily Commuter Data}

For Seoul, we use district-to-district commute flows on the public transit system (subway and bus) through confidential individual trip-level data housed at the Seoul Big Data Campus. Passengers enter and exit public transit using a card with a unique identifier, from which we obtain the time, origin, and destination of each commute. We retain weekdays from $4 \mathrm{am}$ to $12 \mathrm{pm}(12 \mathrm{pm}$ to $8 \mathrm{pm}$ on weekends, since commutes start later) to capture the first commute leg. We aggregate over individual trips to bilateral daily commute flows from January 2018 to April 2020. Pre-pandemic commute flows are the 2018/2019 averages.

For Daegu, we measure daily commuting using subway turnstile data from January 2018 to April 2020, made available by Daegu Metro Transit Corporation. We retain entries and exits using the same commute window as in Seoul. Stations' total entries (exits) plausibly capture the density of residents (jobs) if commuters enter (exit) the subway station closest to their residence (workplace). We aggregate the station-level data to the district level. Pre-pandemic commute flows come from the 2015 Korean Population Census which records where people live and work.

For NYM, we measure county-to-county daily movements using cellphone data from SafeGraph (real-time turnstile data within NYC is not available for suburban commuter rails). The data cover the period from January 1, 2020 to April 30, and are constructed from anonymized smartphone movement data collected daily at the Census block level. ${ }^{9}$ We aggregate these data to the NYM

\footnotetext{
${ }^{9}$ SafeGraph provides the fraction of people who stay at home, which allows us to construct within-district commuting flows.
} 
counties. Pre-pandemic commute flows for NYM are the averages from January 1-20.

Appendix Table A.1 summarizes the commuter data and dates of key events described in the next subsection.

\section{Wages and Population}

Population and wage data for the Korean districts come from the 2019 resident registration database and the 2019 Statistical Yearbook of National Tax. NYM county wages are constructed from the 2017 LEHD Origin-Destination Employment Statistics, which reports the number of workers by wage bin and Census block. ${ }^{10}$ NYM county population in 2019 are from the U.S. Census Bureau.

\section{Credit Card Spending}

We access data on the universe of transactions at brick-and-mortar shops within Seoul using credit and debit cards from Shinhan, one of Korea's top 3 banks. We observe the addresses of cardholders and business, and the purchase values. We construct a daily district-to-district spending matrix from January 2018 to April 2020 among the 25 districts of Seoul, restricting the data to spending by Seoul residents. With this data we estimate elasticities of spending to distance and to post-lockdown commuting flows.

\section{$3.2 \quad$ Commuting Responses}

The first three panels in Figure 1 plot time fixed effects for commuting flows relative to prepandemic averages since January 2020 in each city. For Seoul and NYM, where we observe bilateral

flows, we estimate $\frac{N_{i j t}}{N_{i j, \tau(t)}}=\pi_{t}+\epsilon_{i j t}$. The dependent variable are flows at $t$ relative to pre-pandemic flows and $\tau(t)$ is a day-of-week and month dummy. For Daegu the figure reports $\frac{E_{i t}}{\bar{E}_{i, \tau(t)}}=\pi_{t}+\epsilon_{i t}$, where the dependent variable is daily turnstile entries. The figure reports $\pi_{t}$ for each city.

In each figure the first vertical line denotes the first confirmed case in the country, the middle line denotes the first case in the city, and the last line is the date of the lockdown announcement. We overlay the daily counts of new Covid-19 scaled by city population. ${ }^{11}$

The first confirmed case within Korea occurred on January 26, and ridership in both cities dropped and remained down roughly 10\%. After the first confirmed case within Daegu on February 17, the virus spread quickly and commuting declined steeply. Within Seoul, there was no further change after the first confirmed case on January 30. The right axes show that the spread was much larger in Daegu than in Seoul, which may explain the different commuting responses.

After the virus spread throughout Korea during February, a national task force laid out guidelines that included social distancing, working from home, canceling non-essential gatherings, and postponing the start date for schools and universities. Following the announcement on February

\footnotetext{
${ }^{10}$ Bins are defined as lower than $\$ 1250$, between $\$ 1250$ and $\$ 3333$, and above $\$ 3333$ per month.

${ }^{11}$ Following Fang et al. (2020), we find a positive correlation between lags of commuting and new daily infections after controlling for location and date fixed effects. See Appendix B.
} 
24, ridership fell in both cities for roughly two weeks before slowly trending back upwards. Overall, ridership fell $60.2 \%$ and $34.9 \%$ in Daegu and Seoul, respectively, between the announcement date and April 30. The standard errors suggest similar responses across districts.

In contrast, in NYM we do not observe declines in commuting before the first confirmed withincity case on March 3. New York State issued a lockdown order on March 22 that closed non-essential businesses. ${ }^{12}$ At that time, commuting was already trending downward and continued to fall until mid-April to $70.1 \%$ below the pre-pandemic flows.

\subsection{Model Parameters}

We bring the model to the data using daily frequency. Table 1 summarizes the parameters and Appendix A describes the numerical resolution method.

\section{Disease Dynamics}

Following Ferguson et al. (2020) we set $\gamma_{I}$ consistent with an incubation period of 5.1 days and as robustness also consider 4.2 days (Sanche et al., 2020). Following Wang et al. (2020) we set $\gamma_{R}$ consistent with a recovery time of 18 days and also show results assuming 10 days. Ferguson et al. (2020) obtain an infection fatality ratio of $0.9 \%$, which we use as benchmark, and we also use a lower bound of $0.3 \%$ across studies (Hall et al., 2020). Alamian et al. (2019) estimate that $36 \%$ of cases infections are asymptomatic. We use this number as benchmark and half that rate as robustness. For $\delta_{u}$, Dingel and Neiman (2020) report that $46 \%$ of jobs in the U.S. could be done from home. A survey by Job Korea says that $60 \%$ of workers can telecommute. ${ }^{13}$ The probability of finding a vaccine corresponds to an expected arrival time of 18 months.

The benchmark value of life $\omega$ assumes an expected lifetime of 14.5 years for Covid-19 victims times an annual value of USD 185,000 calculated by Hall et al. (2020) minus the discounted value of wages (already accounted for by the planner in the economic costs). To trace a Pareto frontier we also vary $\omega$ over a range of values between $1 / 100$ and 100 times the benchmark. $\rho$ matches an annual interest rate of $4 \%$.

\section{Matching Function and Transmission rate}

We assume that, given the number of individuals interacting in a location, contagion is more prevalent in denser districts: ${ }^{14}$

$$
\beta_{j}=\frac{\beta}{\text { area }_{j}}
$$

\footnotetext{
${ }^{12}$ New Jersey and Connecticut locked down a few days earlier but we assign the NY's lockdown date.

${ }^{13}$ See http://www . jobkorea.co.kr/GoodJob/Tip/View?News_No=16696publishedMay42020.

${ }^{14}$ This adjustment ensures that the aggregate infections are invariant to spatial aggregation. With $S$ susceptible and $I$ infected individuals equally divided among $J$ locations, assuming away spatial interactions the aggregate number of infections is $\frac{S I}{J}$, so that slicing a territory reduces infections. Normalizing by area yields instead $S I$, which is invariant to $J$.
} 
We set $\beta$ to match the model-based infection dynamics to Covid-19 case data. Using (3) and (16), the change in the number of susceptible agents is:

$$
\Delta S(i, t)=-\beta \zeta\left[\sum_{j} \frac{1}{\operatorname{area}_{j}} \chi(i, j, t) \lambda(i, j) \sum_{i^{\prime}} \chi\left(i^{\prime}, j, t\right) \lambda\left(i^{\prime}, j\right) I\left(i^{\prime}, t\right)\right] S(i, t)+\varepsilon(i, t),
$$

where $\lambda(i, j)$ is the pre-pandemic fraction of residents from $i$ commuting to $j$ at time $t, \chi(i, j, t)$ is the commuting from $i$ to $j$ at time $t$ relative to pre-pandemic flows, and $\varepsilon(i, t)$ accounts for measurement error and other forces driving infections. We observe $\lambda(i, j, t) \chi(i, j, t)$ for Seoul and NYM, and for Daegu we apply the changes in entries and exits to the pre-pandemic flows to construct its bilateral flows. $S(i, t)$ and $I(i, t)$ are recovered from data on new infections, the calibrated transition rates, and the laws of motion (12) to (14). Given the asymptomatic rate $\zeta$, we set $\beta$ to minimize the sum of square errors $\sum_{i} \sum_{t} \varepsilon(i, t)^{2}$.

To mitigate concerns that the new cases data is imprecise and driven by testing, we start the estimation 10 days after the peak in new cases in each city. This approach is consistent with the assumptions that the data on new cases became more precise in the latest periods. The results are very similar if we start the estimation at the peak.

Appendix Figure A.2 shows that the model replicates well the average number of new cases after the peak in the data, and implies a fair amount of dispersion in the dynamics across locations. For the first week after patient zero, the estimation implies a city-level reproduction number (the number of new infections per infected individual) of 1.32 in Seoul, 1.32 in Daegu, and 2.27 in NYM. These numbers are in line with existing estimates; e.g., Shim et al. (2020) estimate 1.5 in Korea and Fernández-Villaverde and Jones (2020) estimate 2.5 in New York. ${ }^{15}$

\section{Trade Model Parameters}

The bottom-right panel of Figure 1 plots $s(i, i, t)$, the share of within-district expenditures, relative to the pre-pandemic levels. Same-district spending shares increase at the time of the lockdown, suggesting that shopping costs increased.

This evidence motivates a specification of trade costs as a function of geographic frictions and lockdown:

$$
\tau(i, j, t)=\kappa_{0} \operatorname{distance}(i, j)^{\kappa_{1}} \chi(i, j, t)^{-\varepsilon},
$$

where $\tau_{0}(i, i) \equiv 1$. From (8), adding a time subscript and an error term we obtain a gravity equation:

$$
\ln X(i, j, t)=\psi(j)+\eta(i)-(\sigma-1) \kappa_{1} \ln (\text { distance }(i, j))+(\sigma-1) \varepsilon \ln (\chi(i, j, t))+\text { error }(i, j, t)
$$

\footnotetext{
${ }^{15}$ The reproduction number is the largest eigenvalue of the matrix $\operatorname{diag}(\mathbf{S}(t)) \mathbf{H}_{S}^{\prime} \operatorname{diag}(\boldsymbol{\beta}) \mathbf{H}_{I} /\left(\gamma_{D}+\gamma_{R}\right)(\operatorname{Diekmann}$ et al., 1990). Table 1 reports the estimates of $\beta$.
} 
where $X(i, j, t)$ are district $j$ 's expenditures in goods and services from $i$ at time $t$, and $\psi(j)$ and $\eta(i)$ are destination and origin fixed effects. ${ }^{16}$

Using the credit-card spending data from Seoul we estimate $(\sigma-1) \kappa_{1}=1.53$ (se 0.066) and $(\sigma-1) \varepsilon=0.45$ (se 0.067). ${ }^{17}$ We set $\sigma=5$ (Ramondo et al., 2016) to recover $\kappa_{1}$ and $\varepsilon$. For each city, we set $z(i)$ and the scale parameter $\kappa_{0}$ to match the pre-pandemic data on wages $w(j)$ and the fraction of same-district expenditures in total expenditures, respectively. The latter is $55 \%$ in the Seoul credit-card data, and assumed to be the same across cities.

\section{Optimal Spatial Lockdowns}

\subsection{Centrality and Optimal Lockdown}

We implement the model in each city using the case distribution at the lockdown date as initial condition. The left panel of Figure 2 shows eigenvector centrality by location, which captures the potential by location to diffuse Covid-19 under no lockdown. ${ }^{18}$ The right panel shows the fraction of commuting inflows that is optimally shut down.

We find similar qualitative patterns in NYM and Daegu. The most central locations first experience a strong lockdown, of up to $60 \%$ in New York and $70 \%$ in Daegu. In these locations the lockdown is only partially relaxed over 3 to 6 months. In NYM, other locations also exhibit an early lockdown but only Manhattan remains closed for a long time. In Daegu, all central locations exhibit strict lockdown for a long time.

These patterns contrast with Seoul, where despite the limited spread the planner imposes a longlasting lockdown to restrain the disease. The planner first locks down a few peripheral locations, but maintains economic activity. After the disease has spread, the lockdown intensifies across more central locations.

The results demonstrate that the optimal strategies over time and space depend on the full geography of commute patterns and real income, and the initial viral spread. When the spread is sufficiently large, the planner first places more weight on shutting down locations that are perceived as transmission hubs, even if they are the main sources of real income. When it is not, those locations are first spared. In either case, the policy maintains a considerable steady-state lockdown to avoid a re-emergence of the disease. ${ }^{19}$

\footnotetext{
${ }^{16}$ Appendix Figure A.1 reports positive correlations between commuting and expenditures relative to the prepandemic levels, confirming a negative impact of lockdown on shopping.

${ }^{17}$ The results are very similar if same-district expenditures are excluded. Monte et al. (2018) estimate a distance elasticity of 1.29 across Commodity Flow Survey regions.

${ }^{18}$ This is the eigenvector associated with the largest eigenvalue of the matrix $\operatorname{diag}(\mathbf{S}(0)) \mathbf{H}_{S}^{\prime} \mathbf{H}_{I}$, weighted by the location-specific transmission rate $\beta_{j}$.

${ }^{19}$ The optimal lockdowns are further visualized in Appendix Figure A.3. The figure shows the lockdown every 30 days. The initial lockdown pattern radiates from geographically central locations and weakens over time.
} 


\subsection{Pareto Frontier: Uniform versus Spatially Optimal Lockdown}

We compute a "Pareto" frontier describing the tradeoff between cases and economic costs. We solve the optimal lockdown for values of life $\omega$ ranging between 1/100 and 100 times the benchmark. To demonstrate the importance of spatially targeted policies, we also implement optimal uniform lockdown paths, restricted to be constant over space, or optimal lockdowns that vary only by origin and destination. Figure 3 plots cumulative Covid-19 cases against the economic loss since the prepandemic period at the last period of our data (April 30) across these values of $\omega$, along with the actual economic costs and cases in the estimated model.

We find large gains from implementing optimal spatial lockdown. Compared to uniform optimization, given the actual number of cases, spatially targeted lockdown leads to $20 \%, 32 \%$, and $55 \%$ lower economic costs in Daegu, Seoul, and NYM, respectively. In NYM and Daegu, the gap in economic cost between uniform and optimal policies grows for higher values of life.

The actual economic costs and cumulative cases were far from the optimal but close to the spaceblind optimal policy. Under spatial targeting, the same number of cumulative cases could have been reached at 19\%,27\%, and 39\% lower economic costs in Daegu, Seoul, and NYM, respectively. The gains from optimal lockdowns by destination are larger than by origin and very close to the benchmark, suggesting that business lockdowns may be enough to reap the benefits of spatial targeting.

\subsection{Optimal and Observed Commuting Reductions}

We now compare observed reductions in commuting with the model's optimal flows. The left panel of Figure 4 shows aggregate commuting relative to pre-pandemic values in the data and under optimal lockdown. Since the optimal policies are implemented at the time of lockdown, they are shown as a flat line until that time. The right panel shows inflows for high and low centrality locations. In Daegu and NYM, the actual city-level reductions in commuting were not very far from the optimal benchmark. In New York, a 40\% drop took place during the time leading up to the lockdown. In the model, the optimal lockdown in that period is about $20 \%$. We find an even closer pattern in Daegu, where the optimal lockdown is weakly relaxed over time, as in the data. However, the most central (peripheral) locations of both NYM and Daegu exhibited a weaker (stronger) reduction in commuting than the optimal. In Seoul, the actual reductions were much stronger than at the optimal in all locations. These differences explain why the estimated economic costs from actual commuting responses were larger than the spatially optimal ones, as in Figure 3.

\subsection{Alternative Specifications}

Appendix Figure A.5 shows that spatial lockdown patterns are robust to the alternative parametrizations described in the previous section. Figure A.6 shows that for a large shock affecting $1 \%$ of the population, or for a value of life equal to 100 times the benchmark, the qualitative patterns in Seoul resemble those in Daegu and NYM. 
We also implement a case where the virus diffuses through shopping. Agents from $i$ now interact with other agents in location $j$ at rate $\chi(i, j, t) \lambda(i, j)+\tilde{\beta} q(i, j, t, \chi(t))$, where $q(i, j, .$.$) is their$ per-capita consumption of goods from $j$ and $\tilde{\beta}$ is the intensity of diffusion through shopping relative to commuting. Table 1 shows the parameter estimates and appendix figures A.7 to A.9 replicate the lockdown policies and Pareto frontier in this case. Compared to the benchmark, the lockdown is weaker in Daegu and Seoul due to the smaller estimate of $\beta$. However, the qualitative spatial patterns of lockdown and the magnitude of the gains from targeted spatial lockdown implied by the Pareto frontier are similar.

\section{Conclusion}

Our framework could be applied to other spatial scales such as across cities, states, or countries, and to the study the optimal spatial deployment of a vaccine in limited supply. Future work could also relax the assumption that worker-job matches are kept constant by allowing for potentially sluggish job reallocations as lockdowns unwind. 


\section{References}

Acemoglu, D., V. Chernozhukov, I. Werning, and M. Whinston (2020). A multi-risk SIR model with optimally targeted lockdown. Technical report, NBER Working Paper 27102.

Adda, J. (2016). Economic activity and the spread of viral diseases: Evidence from high frequency data. The Quarterly Journal of Economics 131(2), 891-941.

Alamian, A., S. Pourbakhsh, A. Shoushtari, and H. Keivanfar (2019). Seroprevalence investigation of newcastle disease in rural poultries of the Northern Provinces (Golestan, Gilan, and Mazandaran) of Iran. Archives of Razi Institute 74(4), 365-373.

Alvarez, F., D. Argente, and F. Lippi (2020). A simple planning problem for COVID-19 lockdown. Technical report, NBER Working Paper 26981.

Anderson, J. E. and E. Van Wincoop (2003). Gravity with gravitas: A solution to the border puzzle. American Economic Review 93(1), 170-192.

Antràs, P., S. J. Redding, and E. Rossi-Hansberg (2020). Globalization and pandemics. Technical report, National Bureau of Economic Research.

Argente, D. O., C.-T. Hsieh, and M. Lee (2020). The cost of privacy: Welfare effect of the disclosure of COVID-19 cases. Technical report, NBER Working Paper 27220.

Arino, J. and P. Van den Driessche (2003). A multi-city epidemic model. Mathematical Population Studies $10(3), 175-193$.

Atkeson, A. (2020a). How deadly is COVID-19? Understanding the difficulties with estimation of its fatality rate. Technical report, NBER Working Paper 26965.

Atkeson, A. (2020b). What will be the economic impact of COVID-19 in the U.S.? Rough estimates of disease scenarios. Technical report, NBER Working Paper 26867.

Baqaee, D. R., E. Fahri, M. Mina, and J. Stock (2020). Reopening scenarios. Technical report, NBER Working Paper 27244.

Birge, J., O. Candogan, and Y. Feng (2020). Controlling epidemic spread: Reducing economic losses with targeted closure. Technical report, University of Chicago.

Bolker, B. and B. T. Grenfell (1995). Space, persistence and dynamics of measles epidemics. Philosophical Transactions of the Royal Society of London. Series B: Biological Sciences 348(1325), 309-320.

Caliendo, L., F. Parro, E. Rossi-Hansberg, and P.-D. Sarte (2018). The impact of regional and sectoral productivity changes on the U.S. economy. The Review of Economic Studies 85(4), 2042-2096.

Cameron, A. C., J. B. Gelbach, and D. L. Miller (2008). Bootstrap-based improvements for inference with clustered errors. Review of Economics and Statistics 90(3), 414-427.

Chinazzi, M., J. T. Davis, M. Ajelli, C. Gioannini, M. Litvinova, S. Merler, A. P. y Piontti, K. Mu, L. Rossi, K. Sun, et al. (2020). The effect of travel restrictions on the spread of the 2019 novel coronavirus (COVID19) outbreak. Science 368(6489), 395-400. 
Diekmann, O., J. A. P. Heesterbeek, and J. A. Metz (1990). On the definition and the computation of the basic reproduction ratio $\mathrm{R} 0$ in models for infectious diseases in heterogeneous populations. Journal of mathematical biology 28(4), 365-382.

Dingel, J. I. and B. Neiman (2020). How many jobs can be done at home? Technical report, NBER Working Paper 26948.

Drakopolous, K. and F. Zheng (2017). Network effects in contagion processes: Identification and control. Technical report, Columbia University.

Eaton, J. and S. Kortum (2002). Technology, geography, and trade. Econometrica 70(5), 1741-1779.

Eubank, S., H. Guclu, V. A. Kumar, M. V. Marathe, A. Srinivasan, Z. Toroczkai, and N. Wang (2004). Modelling disease outbreaks in realistic urban social networks. Nature 429(6988), 180-184.

Fang, H., L. Wang, and Y. Yang (2020). Human mobility restrictions and the spread of the novel coronavirus (2019-nCoV) in china. Technical report, NBER Working Paper 26906.

Ferguson, N., D. Laydon, G. Nedjati Gilani, N. Imai, K. Ainslie, M. Baguelin, S. Bhatia, A. Boonyasiri, Z. Cucunuba Perez, G. Cuomo-Dannenburg, et al. (2020). Report 9: Impact of non-pharmaceutical interventions (NPIs) to reduce COVID-19 mortality and healthcare demand. Technical report, Imperial College.

Fernández-Villaverde, J. and C. I. Jones (2020). Estimating and simulating a SIRD model of COVID-19 for many countries, states, and cities. Technical report, NBER Working Paper 27128.

Flaxman, S., S. Mishra, A. Gandy, H. J. T. Unwin, T. A. Mellan, H. Coupland, C. Whittaker, H. Zhu, T. Berah, J. W. Eaton, et al. (2020). Estimating the effects of non-pharmaceutical interventions on covid-19 in europe. Nature, 1-8.

Germann, T. C., K. Kadau, I. M. Longini, and C. A. Macken (2006). Mitigation strategies for pandemic influenza in the United States. Proceedings of the National Academy of Sciences 103(15), 5935-5940.

Giannone, E., N. Paixao, and X. Pang (2020). The geography of pandemic containment. Technical report, Bank of Canada Working Paper.

Glover, A., J. Heathcote, D. Krueger, and J. V. Rios Rull (2020). Health versus wealth: On the distributional effects of controlling a pandemic.

Goldman, S. M. and J. Lightwood (2002). Cost optimization in the sis model of infectious disease with treatment. Topics in Economic Analysis \& Policy 2(1).

Hall, R. E., C. I. Jones, and P. J. Klenow (2020). Trading off consumption and COVID-19 deaths. Technical report, NBER Working Paper 27340.

Hsiang, S., D. Allen, S. Annan-Phan, K. Bell, I. Bolliger, T. Chong, H. Druckenmiller, L. Y. Huang, A. Hultgren, E. Krasovich, et al. (2020). The effect of large-scale anti-contagion policies on the covid-19 pandemic. Nature, 1-9.

Jones, C., T. Philippon, and V. Venkateswaran (2020). Optimal mitigation policies in a pandemic: Social distancing and working from home. Technical report, NBER Working Paper 26984. 
Kissler, S., N. Kishore, M. Prabhu, D. Goffman, Y. Beilin, R. Landau, C. Gyamfi-Bannerman, B. Bateman, D. Katz, J. Gal, et al. (2020). Reductions in commuting mobility predict geographic differences in SARSCoV-2 prevalence in New York City. Technical report, Harvard University.

Korolev, I. (2020). Quantifying social interactions using smartphone data. Technical report, Binghamton University.

Manski, C. F. and F. Molinari (2020). Estimating the covid-19 infection rate: Anatomy of an inference problem. Journal of Econometrics.

Monte, F., S. J. Redding, and E. Rossi-Hansberg (2018). Commuting, migration, and local employment elasticities. American Economic Review 108(12), 3855-90.

Piguillem, F. and L. Shi (2020). The optimal COVID-19 quarantine and testing policies. Technical report, CEPR Discussion Paper DP14613.

Raifman, J., K. Nocka, D. Jones, J. Bor, S. Lipson, J. Jay, and P. Chan (2020). COVID-19 US state policy database. Technical report, Ann Arbor, MI: Inter-university Consortium for Political and Social Research.

Ramondo, N., A. Rodríguez-Clare, and M. Saborío-Rodríguez (2016). Trade, domestic frictions, and scale effects. American Economic Review 106(10), 3159-84.

Redding, S. J. and E. Rossi-Hansberg (2017). Quantitative spatial economics. Annual Review of Economics 9, 21-58.

Rowthorn, B. R. and F. Toxvaerd (2012). The optimal control of infectious diseases via prevention and treatment. Technical report, CEPR Discussion Paper DP8925.

Rowthorn, R. (2020). A cost-benefit analysis of the COVID-19 disease. CEPR Covid Economics.

Rowthorn, R. and F. Toxvaerd (2020). The optimal control of infectious diseases via prevention and treatment. Cambridge Working Papers in Economics 2027.

Rowthorn, R. E., R. Laxminarayan, and C. A. Gilligan (2009). Optimal control of epidemics in metapopulations. Journal of the Royal Society Interface 6(41), 1135-1144.

Rvachev, L. A. and I. M. Longini Jr (1985). A mathematical model for the global spread of influenza. Mathematical biosciences 75(1), 3-22.

Sanche, S., Y. T. Lin, C. Xu, E. Romero-Severson, N. Hengartner, and R. Ke (2020). Early release-high contagiousness and rapid spread of severe acute respiratory syndrome coronavirus 2. Emerging Infectious Diseases 26, 1470-1477.

Shim, E., A. Tariq, W. Choi, Y. Lee, and G. Chowell (2020, apr). Transmission potential and severity of COVID-19 in south korea. International Journal of Infectious Diseases 93, 339-344.

Stock, J. H. (2020). Data gaps and the policy response to the novel coronavirus. Technical report, NBER Working Paper 26902.

Tian, H., Y. Liu, Y. Li, C.-H. Wu, B. Chen, M. U. Kraemer, B. Li, J. Cai, B. Xu, Q. Yang, et al. (2020). An investigation of transmission control measures during the first 50 days of the COVID-19 epidemic in china. Science 368(6491), 638-642. 
Viboud, C., O. N. Bjørnstad, D. L. Smith, L. Simonsen, M. A. Miller, and B. T. Grenfell (2006). Synchrony, waves, and spatial hierarchies in the spread of influenza. Science 312(5772), 447-451.

Wang, H., Z. Wang, Y. Dong, R. Chang, C. Xu, X. Yu, S. Zhang, L. Tsamlag, M. Shang, J. Huang, et al. (2020). Phase-adjusted estimation of the number of coronavirus disease 2019 cases in Wuhan, China. Cell discovery $6(1), 1-8$. 
Figure 1: Commute and Spending Responses and Disease Spread
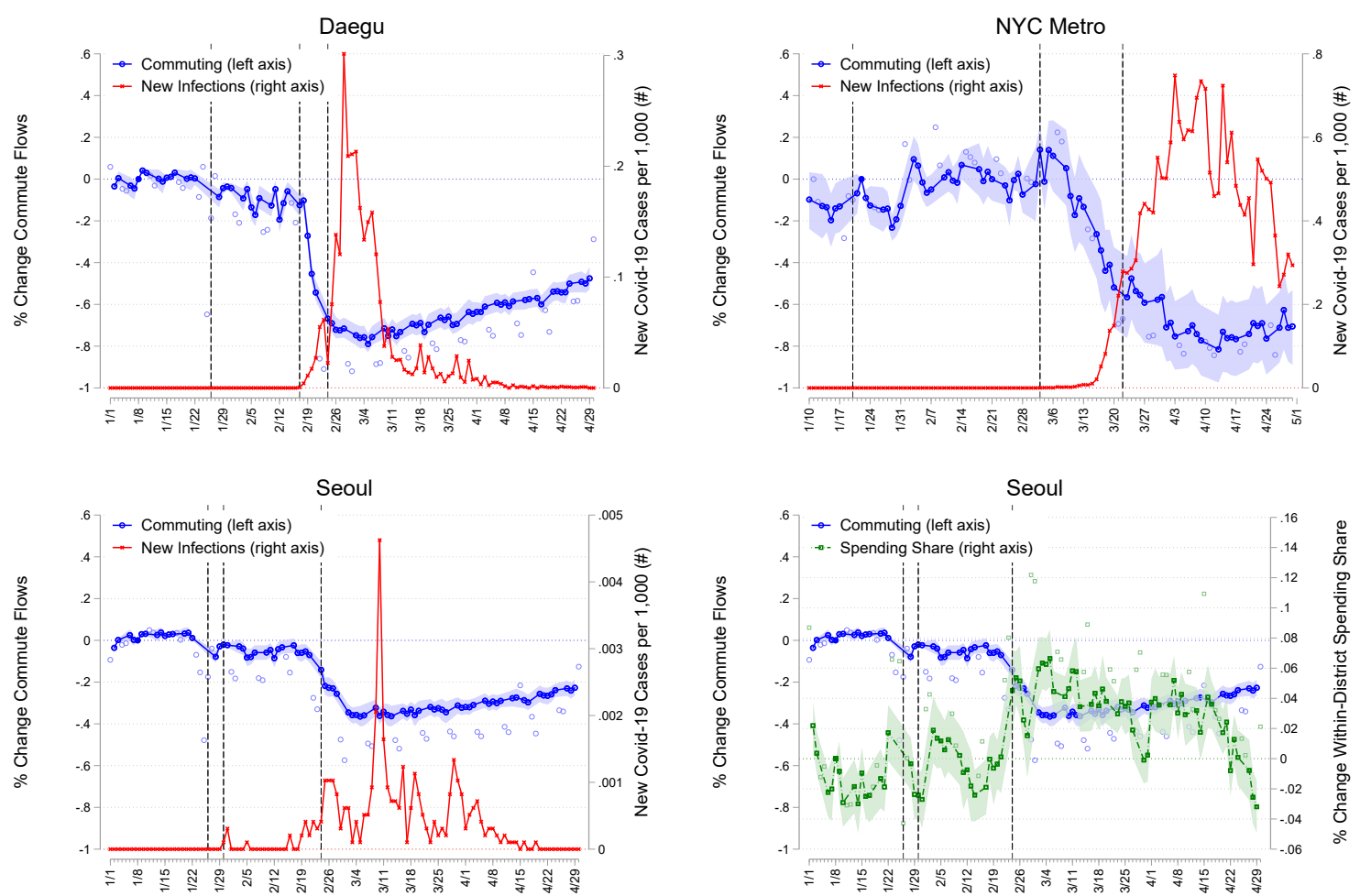

Note: Figure reports the average daily changes in commute flows relative to the pre-pandemic levels, corresponding to the time fixed effects from the equation in Section 3.2 (left axis). The time fixed effects are normalized to 0 on January 8, 2020 for Daegu and Seoul (January 22, 2020 for NYM). Weekdays (weekends/holidays) are denoted in darker (lighter) circles. The regression sample size is 968 for Daegu, 75,625 for Seoul, and 46,325 for NYM. Each observation is a district by date pair for Daegu and a district/county-pair by date tuple for Seoul and NYM from January 1, 2020 to April 30. The first vertical line in each graph denotes the date of the first case in the country. The middle and last vertical lines denote the date of the first case and the lockdown announcement in each city, respectively. Wild bootstrap standard errors are clustered by district for Daegu, and two way-clustered by origin and destination for Seoul and NYM (Cameron et al., 2008). Error bars show 95\% confidence intervals. The right axis reports the daily new Covid-19 cases in Daegu (top left), NYM (top right), and Seoul (bottom left). The right axis in the bottom-right panel reports the average daily changes in the share of each district's expenditures spent in the same district relative to the pre-pandemic levels, denoted in squares (the commuting response in the bottom right panel is replicated from the bottom left panel). 


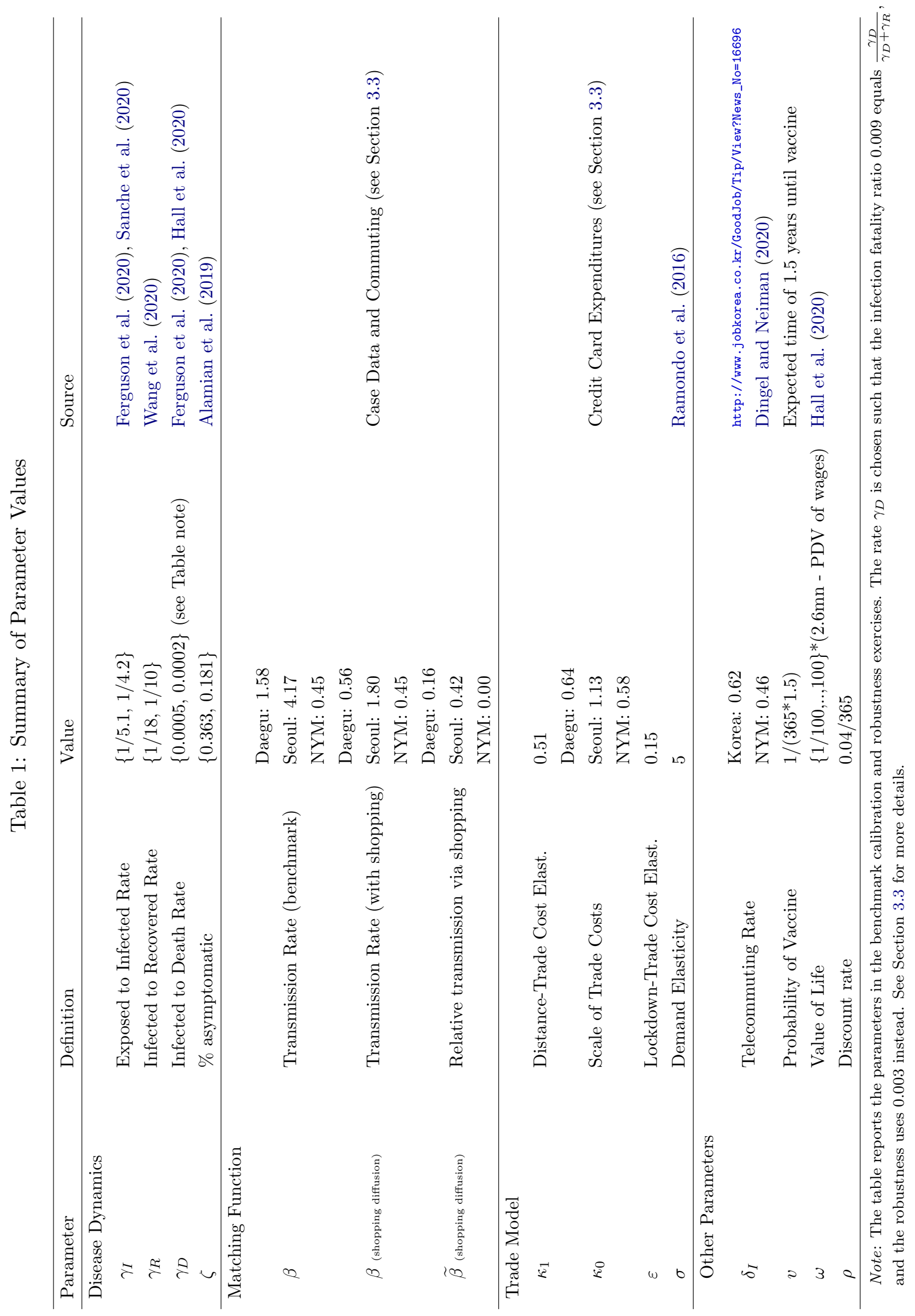


Figure 2: Centrality of Commuting Locations and Optimal Policies

(a) Daegu
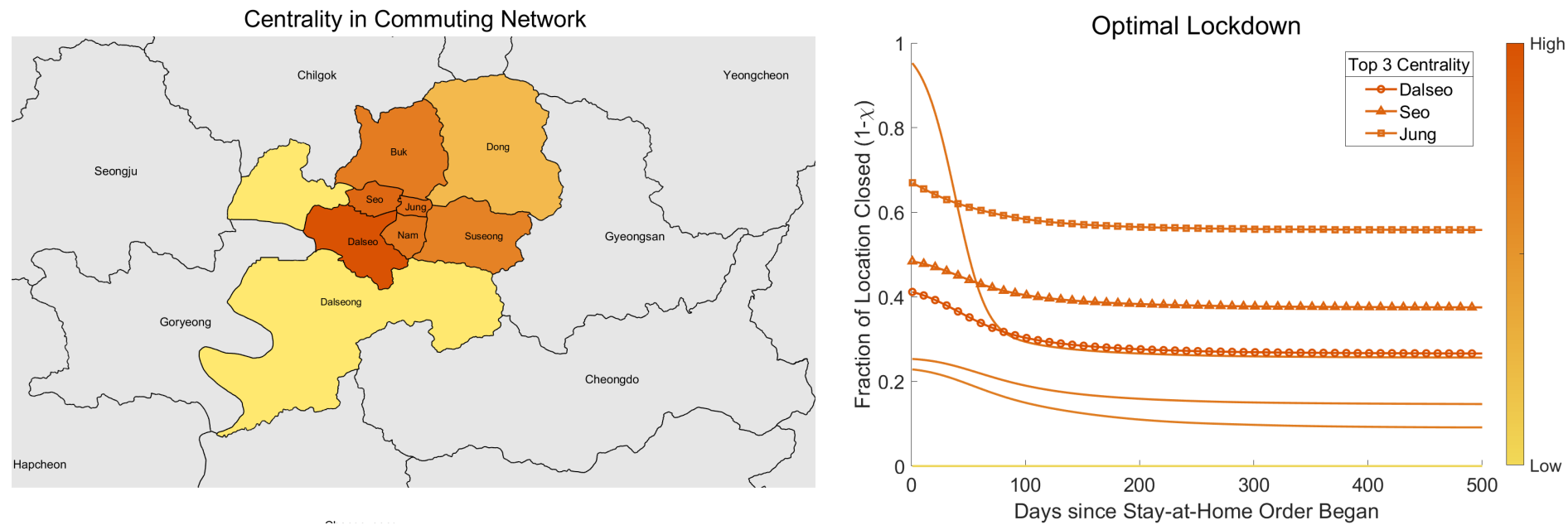

(b) Seoul
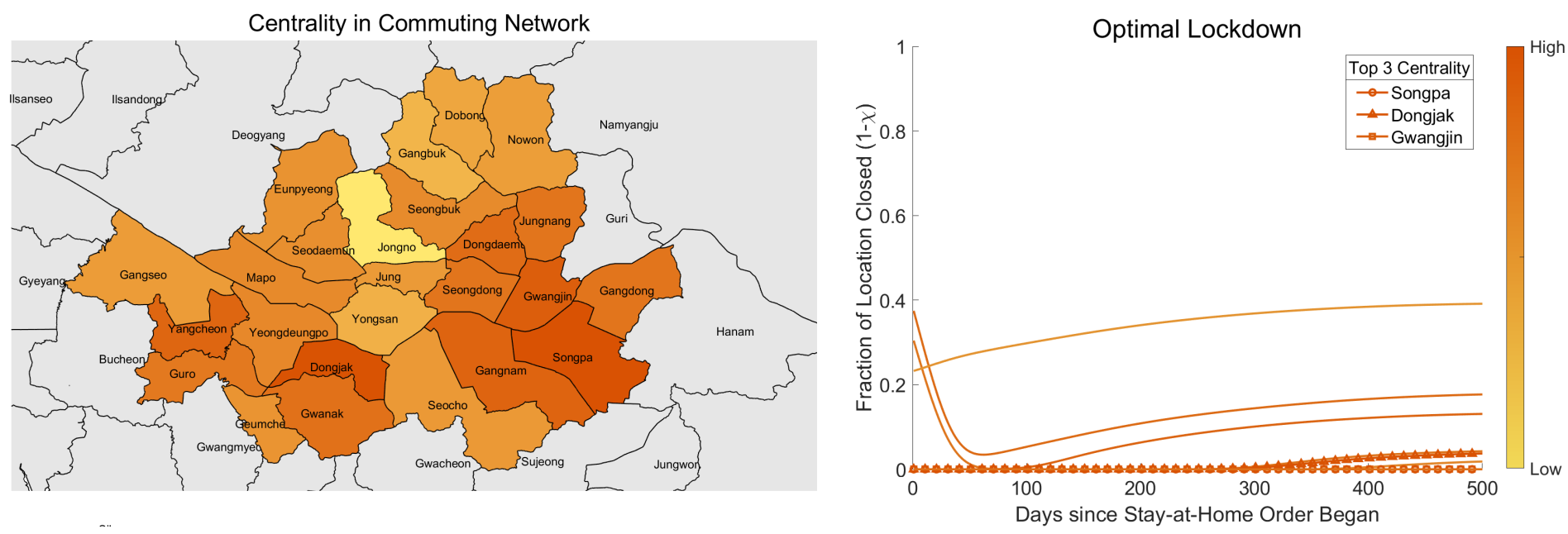

(c) NYC Metro
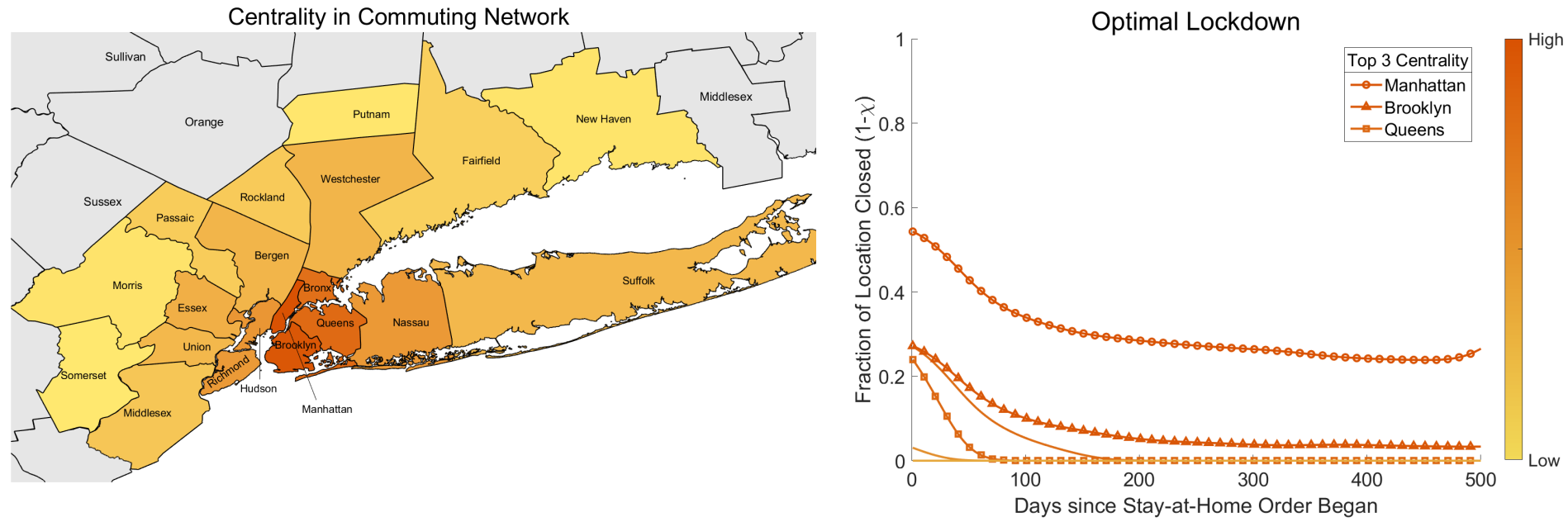

Note: The left panel denotes the (log) centrality of a location (see footnote 18), normalized so that the most central location is 1 . The right panel plots the optimal policies over time for each location in the network. The color of the line represents the centrality of the location in the network. The three most central locations in the network are indicated in the legend. 
Figure 3: Pareto Frontiers

(a) Daegu

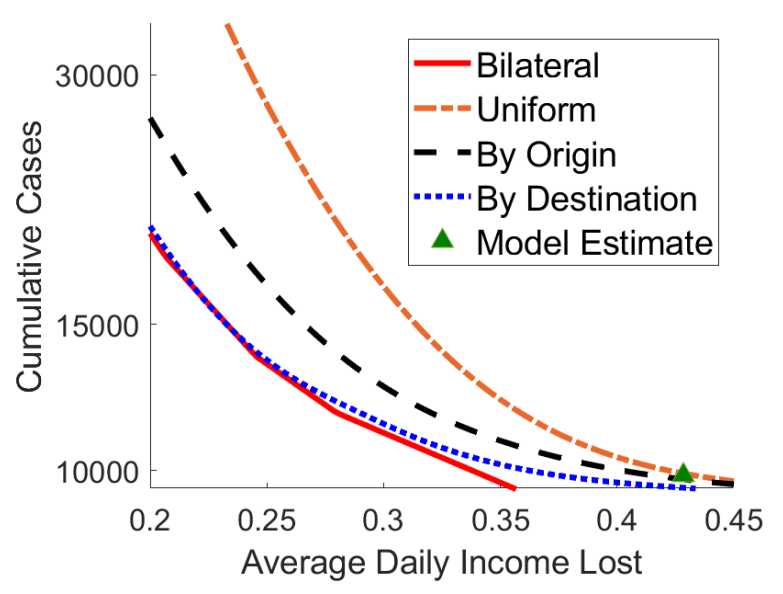

(b) Seoul

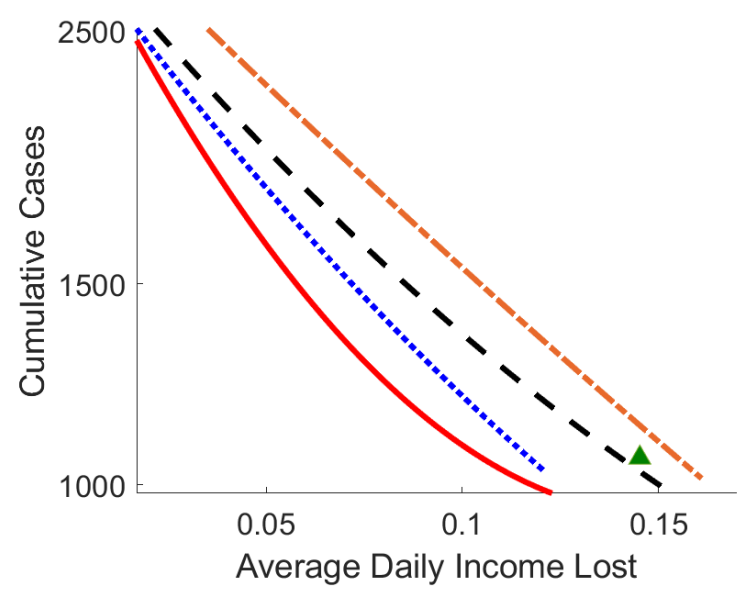

(c) NYC Metro

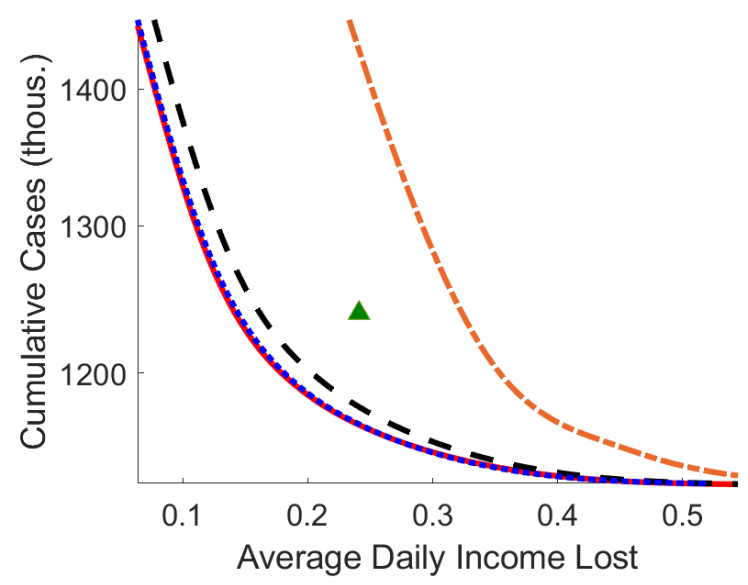

Note: The figures plot the cumulative number of new cases (y-axis, log scale) and the average real income lost per day between the date of the first confirmed case (see Appendix Table A.1) and April 302020 for parametrizations of the value of life $(\omega)$ ranging from 1/100 to 100 times the benchmark, in both the optimal lockdown with space and time variation ("Bilateral"), in the spatially uniform optimal solution with time variation only ("Uniform", the same lockdown across all locations), and in the optimal solution where only origin or destination can be closed ("by Origin" and "by Destination", respectively). The green triangle shows the case count and real income lost implied by the estimated model on April 302020. 
Figure 4: Changes in Commuting Flows: Optimal and Observed

(a) Daegu
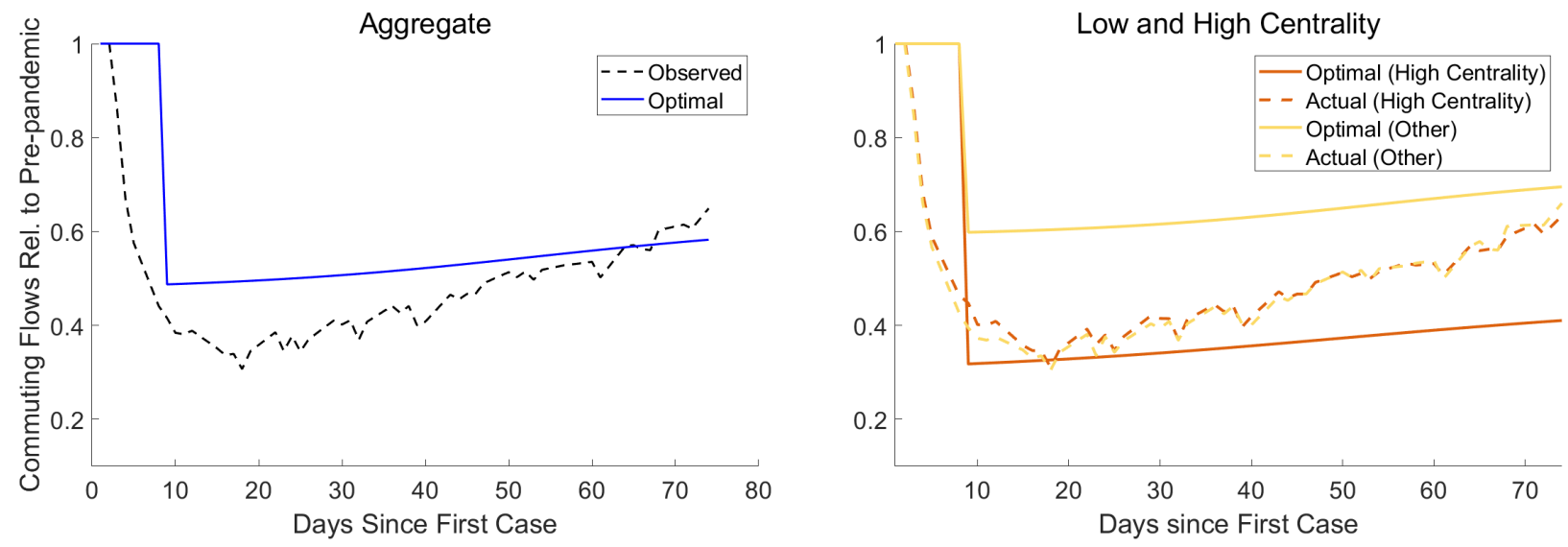

(b) Seoul
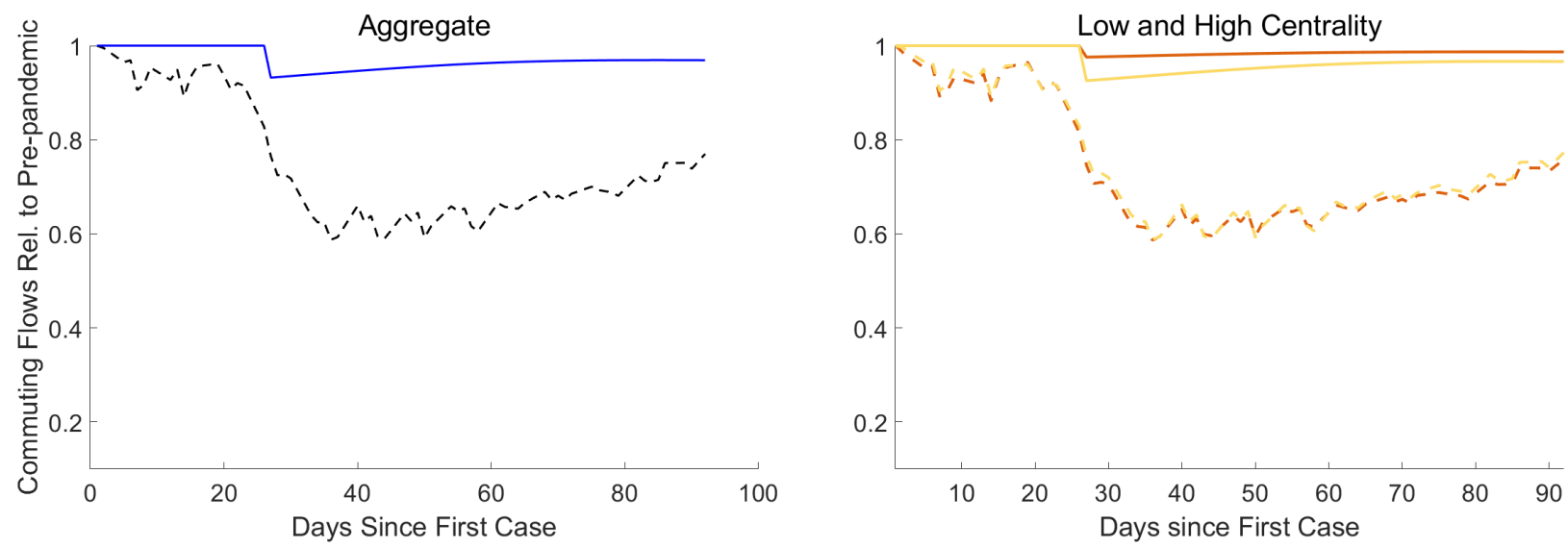

(c) NYC Metro
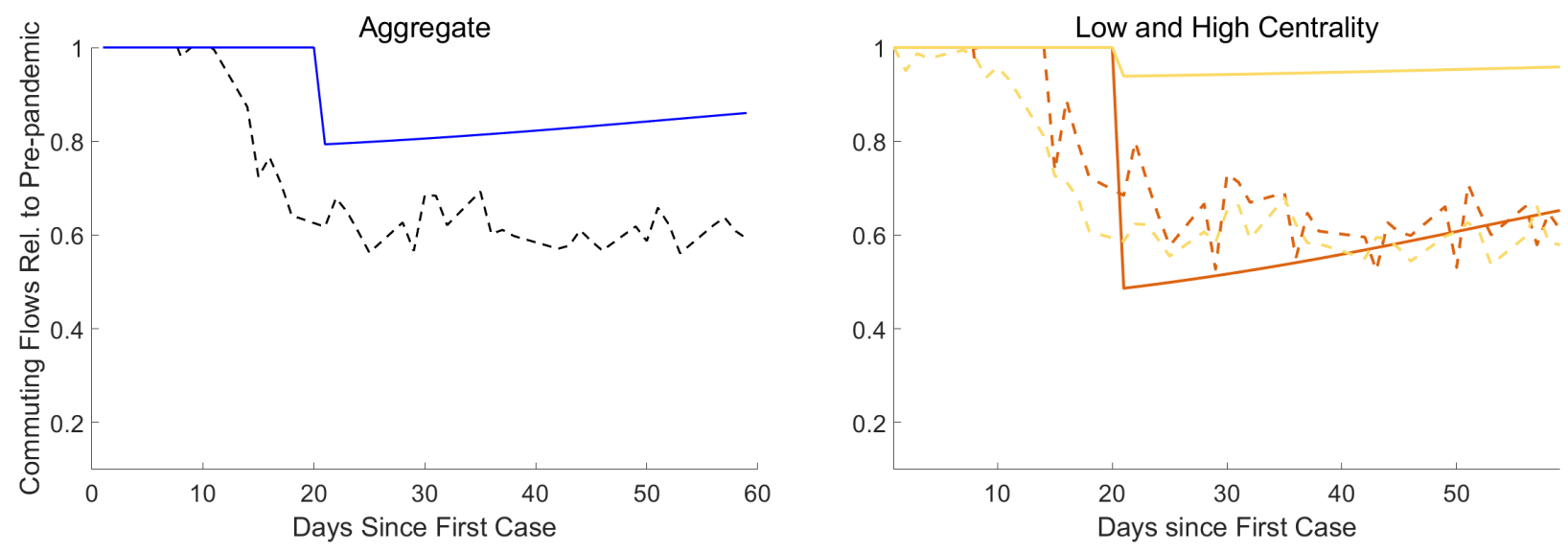

Note: In the left panel, the dashed black line shows the aggregate commuting flows in each city starting from the date of the first confirmed case in each city. The solid and circled blue lines show the aggregate commuting flows implied by the optimal spatial policy. In the right panel, optimal and observed commuting responses are divided by top-3 centrality locations (darker shade) and the other locations. 


\section{Online Appendix}

\section{A Numerical Implementation}

This section describes how the optimal planning problem is numerically implemented.

\section{A.1 Optimal control problem}

Assuming that the matching function is $M_{j}(\tilde{I}, \tilde{S})=\beta_{j} \tilde{I} \tilde{S}$, the optimal control problem (11) simplifies to

$$
W=\max _{\chi(t)} \int_{0}^{\infty} e^{-(r+\nu) t} \sum_{j}\left[U(j, t)+\frac{\nu}{r} \bar{U}(j, t)-\omega \gamma_{D} I(j, t)\right] d t
$$

subject to

$$
\begin{aligned}
\dot{\mathbf{S}}(t) & =-\mathbf{S}(t) \cdot\left[\mathbf{H}_{S}(t)^{\prime} \operatorname{diag}(\boldsymbol{\beta}) \mathbf{H}_{I}(t) \mathbf{I}(t)\right] \\
\dot{\mathbf{E}}(t) & =-\dot{\mathbf{S}}(t)-\gamma_{I} \mathbf{E}(t) \\
\dot{\mathbf{I}}(t) & =\gamma_{I} \mathbf{E}(t)-\left(\gamma_{R}+\gamma_{D}\right) \mathbf{I}(t) \\
\dot{\mathbf{R}}(t) & =\gamma_{R} \mathbf{I}(t)
\end{aligned}
$$

and

$$
\begin{aligned}
& U(j, t)=U(j ; \mathbf{S}(t), \mathbf{E}(t), \mathbf{I}(t), \mathbf{R}(t), \boldsymbol{\chi}(t)) \\
& \bar{U}(j, t)=U(j ; 0,0,0, \mathbf{S}(t)+\mathbf{E}(t)+\mathbf{I}(t)+\mathbf{R}(t)) .
\end{aligned}
$$

The present-value hamiltonian can be written

$$
\begin{aligned}
H(t) & =\sum_{j}\left[U(j, t)+\frac{\nu}{r} \bar{U}(j, t)-\omega \gamma_{D} I(j, t)\right. \\
& \left.+\mu_{S}(j, t) \dot{\mathbf{S}}(j, t)+\mu_{E}(j, t) \dot{\mathbf{E}}(j, t)+\mu_{I}(j, t) \dot{\mathbf{I}}(j, t)+\mu_{R}(j, t) \gamma_{R} \mathbf{I}(j, t)\right],
\end{aligned}
$$

where $\boldsymbol{\mu}_{u}, u=S, E, I, R$, are $J \times 1$ vectors of costate variables associated to each sickness status.

The first-order conditions of the problem are

$$
\begin{aligned}
& {[\mathbf{S}(t)] \quad\left(D_{\mathbf{S}} \mathbf{U}(t)+\frac{\nu}{r} D_{\mathbf{R}} \overline{\mathbf{U}}(t)\right)^{\prime} \mathbf{1}_{J \times 1}+\operatorname{diag}\left(\mathbf{H}_{S}(t)^{\prime} \operatorname{diag}(\boldsymbol{\beta}) \mathbf{H}_{I}(t) \mathbf{I}(t)\right)\left(\boldsymbol{\mu}_{E}(t)-\boldsymbol{\mu}_{S}(t)\right) } \\
&=-\dot{\boldsymbol{\mu}}_{S}(t)+(r+\nu) \boldsymbol{\mu}_{S}(t) \\
& {[\mathbf{E}(t)] \quad\left(D_{\mathbf{E}} \mathbf{U}(t)+\frac{\nu}{r} D_{\mathbf{R}} \overline{\mathbf{U}}(t)\right)^{\prime} \mathbf{1}_{J \times 1}+\gamma_{I}\left(\boldsymbol{\mu}_{I}(t)-\boldsymbol{\mu}_{E}(t)\right)=-\dot{\boldsymbol{\mu}}_{E}(t)+(r+\nu) \boldsymbol{\mu}_{E}(t) } \\
& {[\mathbf{I}(t)] \quad\left(D_{\mathbf{I}} \mathbf{U}(t)+\frac{\nu}{r} D_{\mathbf{R}} \overline{\mathbf{U}}(t)\right)^{\prime} \mathbf{1}_{J \times 1}-\omega \gamma_{D} \mathbf{1}_{J \times 1} } \\
&+\mathbf{H}_{I}(t)^{\prime} \operatorname{diag}(\boldsymbol{\beta}) \mathbf{H}_{S}(t) \operatorname{diag}(\mathbf{S}(t))\left(\boldsymbol{\mu}_{E}(t)-\boldsymbol{\mu}_{S}(t)\right) \\
&-\left(\gamma_{R}+\gamma_{D}\right) \boldsymbol{\mu}_{I}(t)+\gamma_{R} \boldsymbol{\mu}_{R}(t)=-\dot{\boldsymbol{\mu}}_{I}(t)+(r+\nu) \boldsymbol{\mu}_{I}(t) \\
& {[\mathbf{R}(t)] }\left(D_{\mathbf{R}} \mathbf{U}(t)+\frac{\nu}{r} D_{\mathbf{R}} \overline{\mathbf{U}}(t)\right)^{\prime} \mathbf{1}_{J \times 1}=-\dot{\boldsymbol{\mu}}_{R}(t)+(r+\nu) \boldsymbol{\mu}_{R}(t) \\
& {\left[\chi_{t}(j, k)\right] } \frac{\partial H(t)}{\partial \chi_{t}(j, k)}=0
\end{aligned}
$$




\section{A.2 Algorithm}

We solve the optimal control problem using the following steps. Set the terminal period $T$ to be a large number. Given some initial condition $\left\{\mathbf{S}^{(n)}(0), \mathbf{E}^{(n)}(0), \mathbf{I}^{(n)}(0), \mathbf{R}^{(n)}(0), \mathbf{D}^{(n)}(0)\right\}$,

1. Initialize $n:=1$. Guess the policy $\chi^{(1)}(t)$ for $t=0 \ldots T$ at the first iteration.

2. Using $\chi^{(n)}(t)$, solve the partial differential equations (A.1)-(A.4) forward using the Euler method to recover $\left\{\mathbf{S}^{(n)}(t), \mathbf{E}^{(n)}(t), \mathbf{I}^{(n)}(t), \mathbf{R}^{(n)}(t), \mathbf{D}^{(n)}(t)\right\}$ for $t=1 \ldots T$. Solve for the economic allocation and the corresponding Jacobian in each $t$ as described in the subsection A.3.

3. Using $\chi^{(n)}(t)$ and the disease states, solve the partial differential equations (A.5)-(A.8) of the costates $\left\{\boldsymbol{\mu}_{S}(t), \boldsymbol{\mu}_{E}(t), \boldsymbol{\mu}_{I}(t), \boldsymbol{\mu}_{R}(t)\right\}_{t=0}^{T-1}$ backward using the Euler method with terminal condition

$$
\left\{\boldsymbol{\mu}_{S}(T), \boldsymbol{\mu}_{E}(T), \boldsymbol{\mu}_{I}(T), \boldsymbol{\mu}_{R}(T)\right\}=0
$$

4. Compute $\chi^{*}(t)=\operatorname{argmax}_{\chi} H^{(n)}(t ; \chi)$ using a numerical optimizer. This step uses the analytical gradient for the trade model described in the next section.

5. Stop if $\left\|\chi^{(n)}-\chi^{*}\right\|<\varepsilon$. Otherwise, set $\chi^{(n+1)}=\lambda \chi^{*}+(1-\lambda) \chi^{(n)}$ where $0<\lambda<1$, set $n:=n+1$ and return to step (2).

Computing the maximizer $\chi^{*}(t)$ for all $t$ is the most computationally expensive step. Computation times can be improved by sampling a smaller number of dates $t_{1}, t_{2} \ldots t_{N}$ and interpolating the policy between those dates.

\section{A.3 Solving the General Equilibrium Trade Model}

We use two methods to compute the solution of the general equilibrium trade model at different stages of the numerical optimization:

\section{Exact Solution}

When solving the SEIR model forward, we compute the exact general equilibrium solution of the trade model by iterating over $w(j, t)$ for each $t$ on the goods market equilibrium equation given a distribution of the state variables. Specifically, combining (4) and (7) we obtain:

$$
w(j, t)=\frac{\sum_{k} Y(k, t)\left(\frac{\tau(j, k, t)}{P(k, t)} \frac{w(j, t)}{z(j)}\right)^{1-\sigma}}{\sum_{u=S, E, I, R} \sum_{k} N_{u}(k, j, t)} .
$$

Further using (5) and (9) we obtain a system for wages at time $t$ of the form

$$
w(j, t)=H_{j}(w(1, t), . ., w(J, t), t)
$$

where the operator $H_{j}\left(w_{1}, . ., w_{J}\right)$ takes the form

$$
H_{j}\left(w_{1}, . ., w_{J}\right)=\left(\frac{1}{\sum_{u=S, E, I, R} \sum_{k^{\prime}} N_{u}\left(k^{\prime}, j, t\right)} \sum_{k} \frac{\left(\frac{\tau(j, k, t)}{z(j)}\right)^{1-\sigma}}{\sum_{i}\left(\frac{\tau(i, k, t)}{z(i)} w_{i}\right)^{1-\sigma}} \sum_{u=S, E, I, R} \sum_{i^{\prime}} N_{u}\left(k, i^{\prime}, t\right) w_{k}\right)^{\frac{1}{\sigma}}
$$

where $N_{u}(i, j, t)$ is given by $(6)$.

\section{Gradients}

When evaluating the Jacobians $D_{u} \mathbf{U}(t)$ and $D_{u} \overline{\mathbf{U}}(t)$ for $u=S, E, I, R$ or when maximizing the Hamiltonian, we linearize the trade model around a nonlinear solution (the equilibrium under the current $\chi$ to evaluate the Jacobian 
and the current equilibrium with $\chi=1$ for the Hamiltonian maximization).

Solving for the linearized equilibrium solely requires inverting a matrix for which we have an analytical expression. Specifically, totally differentiating the equilibrium conditions given shocks to the bilateral flows $d \ln N_{u}(j, i)$ of type- $u$ workers or changes in trade $\operatorname{costs} d \ln \tau$, and dropping the time subscript to save notation, we obtain the following linear system

$$
\begin{aligned}
d \ln Y & =\sum_{u}\left(s_{R}(u) \cdot d \ln N_{u}\right) 1_{J 1}+\sum_{u} s_{R}(u) d \ln w \\
d \ln w & =s_{X} d \ln Y-\left(s_{X} \cdot d \ln s_{M}\right) 1_{J 1}-\sum_{u}\left(s_{W}(u) \cdot d \ln N_{u}\right)^{\prime} 1_{J 1} \\
d \ln P & =s_{M}^{\prime} d \ln w+\left(s_{M} \cdot d \ln \tau\right) 1_{J 1} \\
d \ln s_{M} & =(1-\sigma) d \ln w \times 1_{1 J}-(1-\sigma) 1_{J 1} d \ln P^{\prime}+(1-\sigma) d \ln \tau
\end{aligned}
$$

where the first line is the total differential of (5), the second line corresponds to (7), the third line differentiates the price index (9) and the last line is the changes in the expenditure share, and where we are using vector notation such that $\left[d \ln N_{u}\right]_{i, j}=N_{u}(i, j),[d \ln Y]_{j}=d \ln Y(j),[d \ln P]_{j}=d \ln P(j),[d \ln w]_{j}=d \ln w(j)$, and $\left[s_{M}\right]_{i j}=s(i, j)$ is the expenditure share. In these expressions, we have also defined $\left[s_{X}\right]_{i j}=s_{X}(i, j),\left[s_{W}(u)\right]_{i j}=s_{W}(u, i, j)$ and $\left[s_{R}(u)\right]_{i j}=s_{R}(u, i, j)$ such that $s_{X}(i, j) \equiv \frac{Y(j) s(i, j)}{w(i) \sum_{u=S, E, I, R} \sum_{j} N_{u}(j, i)}$ is location $j$ share in $i$ 's sales, $s_{W}(u, i, j)=$ $\frac{N_{u}(i, j)}{\sum_{u=S, E, I, R} \sum_{k} N_{u}(k, j)}$ is the fraction of $j$ 's efficiency units corresponding to type $u$ commuters from $i$, and $s_{R}(u, i, j)=$ $\frac{N_{u}(j, i) w(i)}{Y(j)}$ is the fraction of resident of $j$ 's income corresponding to commuters to $i$ in type $u$.

We can summarize the expressions as a solution for wages as a function of expenditure shares and labor flows shares:

$$
\begin{aligned}
d \ln w=\Omega_{w}^{-1} & {\left[\sum_{u} s_{X}\left(s_{R}(u) \cdot d \ln N_{u}\right) 1_{J 1}-(1-\sigma) s_{X}\left(s_{M} \cdot d \ln \tau\right)^{\prime} 1_{J 1}\right.} \\
& \left.+(1-\sigma)\left(s_{X} \cdot d \ln \tau\right) 1_{J 1}-\sum_{u}\left(s_{W}(u) \cdot d \ln N_{u}\right)^{\prime} 1_{J 1}\right]
\end{aligned}
$$

where

$$
\Omega_{w}=I_{J}-\sum_{u} s_{X} s_{R}(u)-(1-\sigma) \operatorname{diag}\left(s_{X} 1_{J 1}\right)+(1-\sigma) s_{X} s_{M}^{\prime}
$$

The gradients with respect to $\chi, S, E, I$ and $R$ then follow using the definition of $N_{u}(i, j)$ in $(6)$.

\section{B Relationship between Commuting and Infections}

We follow Fang et al. (2020) to assess the relationship between new daily infections and 21-day lags of commuting in a reduced form specification:

$$
\ln \left(1+\text { new } \operatorname{cases}_{i t}\right)=\alpha_{i}+\gamma_{c i t y(i), t}+\sum_{k=0}^{21} \beta_{k} \ln \left(\text { flow }_{i, t-k}\right)+\epsilon_{i t}
$$

where $\alpha_{i}$ is a district fixed effect and $\gamma_{c i t y(i), t}$ is a fixed effect that varies by city and date $t$. This specification flexibly controls for city-level trends due to forces other than commuting. It identifies the impact of flows from cross-sectional variation by exploiting a district's flows above or below its average. The variable flowit is either the total number of people who leave from district $i$ (outflows) or arrive into district $i$ (inflows). The specification pools over the three cities since number of districts in each city is small, but we weight the regression so that each city contributes equally. Standard errors are clustered by $i$ using the block bootstrap to account for a small number of clusters (Cameron et al., 2008).

We do not have an instrument for commuting that varies across space, so the coefficients must be interpreted with caution. For example, changes in commuting may be correlated with behavioral changes. Figure A.4 reports 
the coefficients. It shows an inverted-U shape peaking between 8-15 days when looking at inflows. The p-value of the joint test $\beta_{k}=0$ for $k=\{0, \ldots, 7\}$ is 0.502 , for $k=\{8, \ldots 15\}$ is 0.028 , and for $k=\{16, \ldots, 21\}$ is 0.461 . The results for outflows are noisier and we do not target these moments in the structural estimation, but they seem consistent with an incubation period after which people showing symptoms get tested or come to the hospital.

\section{Robustness of Optimal Lockdown Patterns}

We implemented robustness with respect to key parameters, as described in Section 3.3. We consider a lower infection fatality ratio $(0.03 \%)$; a faster recovery time (10 days); an estimation of the transmission rate starting at the peak of new cases; an asymptomatic rate of half the benchmark; a large shock such that $1 \%$ of the population is infected; twice the value of life of the benchmark; and a shorter incubation (4.1 days). Figure A.5 shows that the qualitative patterns of optimal lockdown from the benchmark are similar across these specifications, except for Seoul given a large shock, as previously mentioned. Doubling the value of life or introducing a large shock leads to stronger initial lockdown, in particular for central locations, while a lower death rate weakens it. 


\section{Additional Tables and Figures}

Table A.1: Commuter Data Summary Statistics

\begin{tabular}{lccc}
\hline & Daegu & Seoul & NYC Metro \\
\hline Population & $2,438,031$ & $9,729,107$ & $19,467,622$ \\
\# Districts & 8 & 25 & 20 \\
Sample Period & Jan 1, 2018-Apr 30,2020 & Jan 1, 2018-Apr 30, 2020 & Jan 1, 2020-Apr 30,2020 \\
Data Source & Subway ridership & Subway/bus ridership & Mobile phones \\
Flow Type & Turnstile & Bilateral & Bilateral \\
First Case & Feb 17, 2020 & Jan 30, 2020 & Mar 3, 2020 \\
Lockdown Date & Feb 24, 2020 & Feb 24, 2020 & Mar 22, 2020 \\
\# Cumulative Cases & 6,778 & 354 & 389,603 \\
\hline
\end{tabular}

Notes: Table reports summary statistics for the Daegu, Seoul, and NYC Metro data. Administrative units within the two Korean cities are called districts with an average population of 368,701 and an average land area of $45 \mathrm{~km}^{2}$. Administrative units within NYC Metro are counties with an average population of 1,232,768 and an average land area of $690 \mathrm{~km}^{2}$. Cumulative Covid-19 cases are as of April 302020. 
Figure A.1: Bilateral Commute Flows and Expenditures
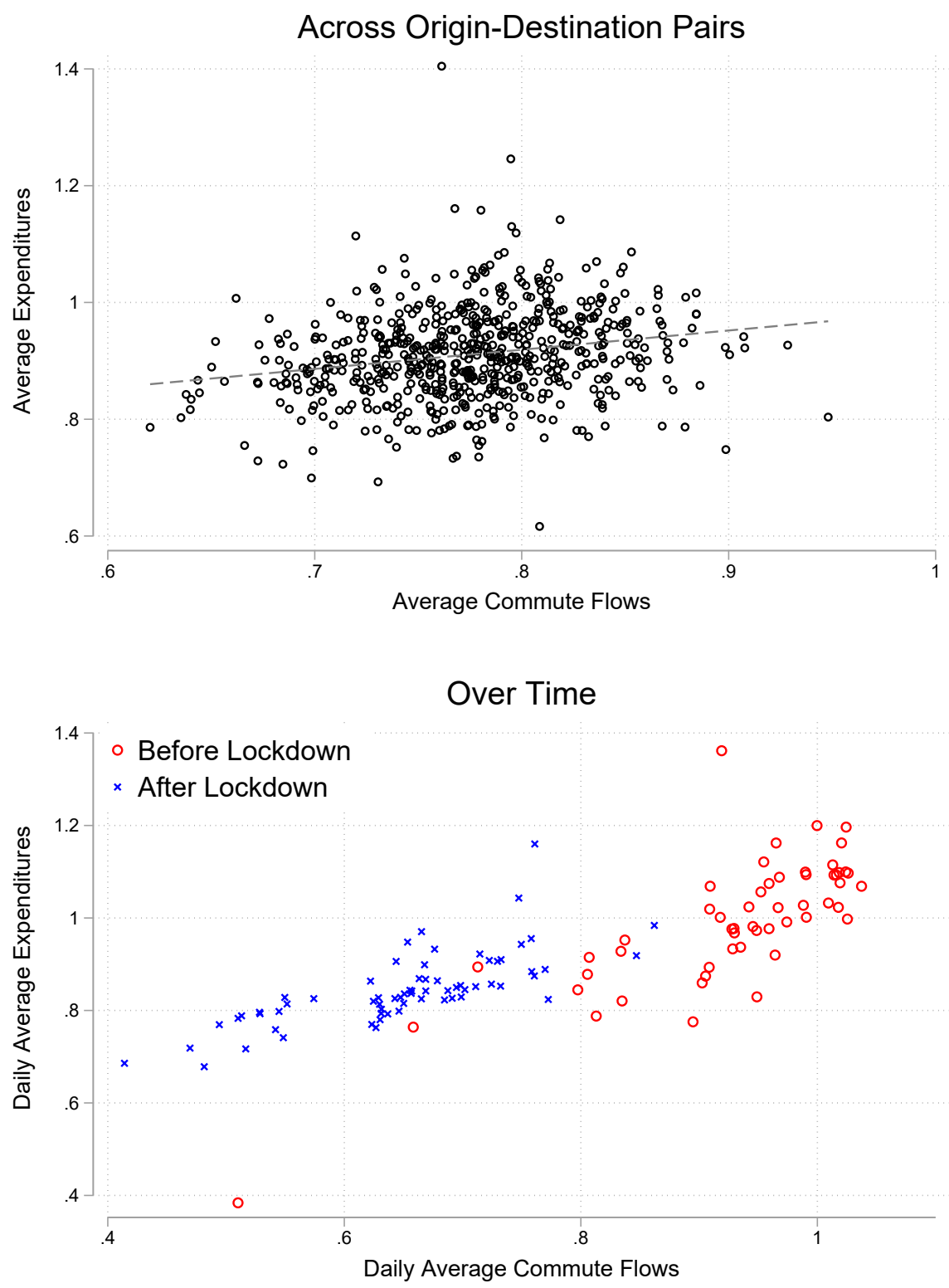

Note: Figure reports district-to-district expenditures against commute flows for Seoul, both normalized by their pre-pandemic levels. The top panel reports commute flows and expenditures for each of the 625 bilateral district pairs averaged over January 12020 to April 30 2020. The bottom panel reports commute flows and expenditures averaged across all 625 district pairs for each of the 121 days during this time period. 
Figure A.2: New Cases per District: Data and Estimation

(a) Daegu

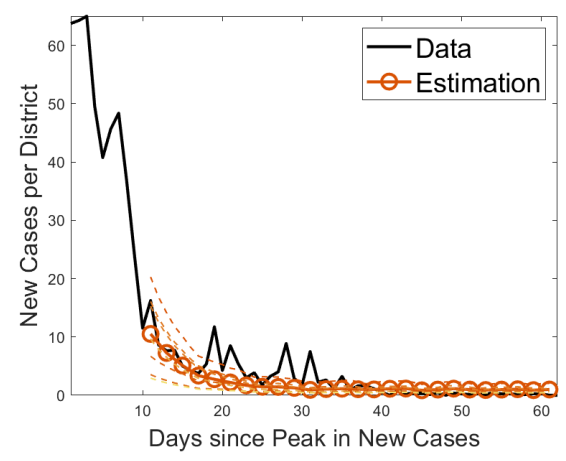

(b) Seoul

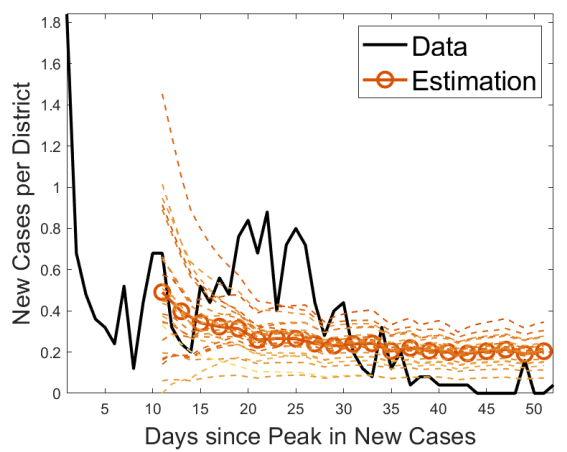

(c) NYC Metro

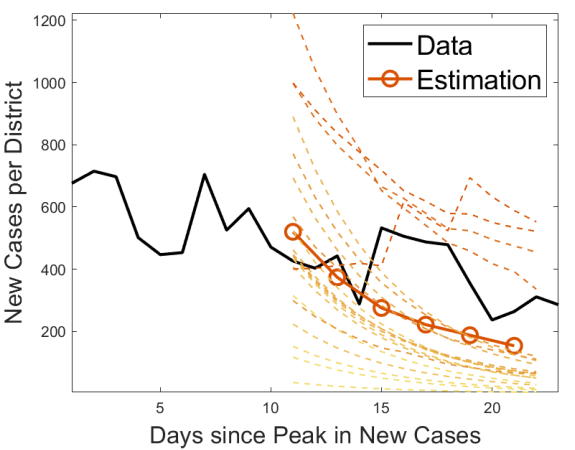

Note: The solid lines show the average number of new cases per district over time since the peak in new cases in the data. The dashed lines are the number of new cases by district in the estimated model corresponding to equation (18), assuming that commuting changed as observed in the data (the shade of the lines represent the share of commuter inflows, with darker shades representing more inflows). The calibration is implemented using case data starting 10 days after the peak in new cases in each city. The solid line with circle markers is the total case number per district in the estimated model. 
Figure A.3: Optimal Lockdown over Districts and Time

Daegu
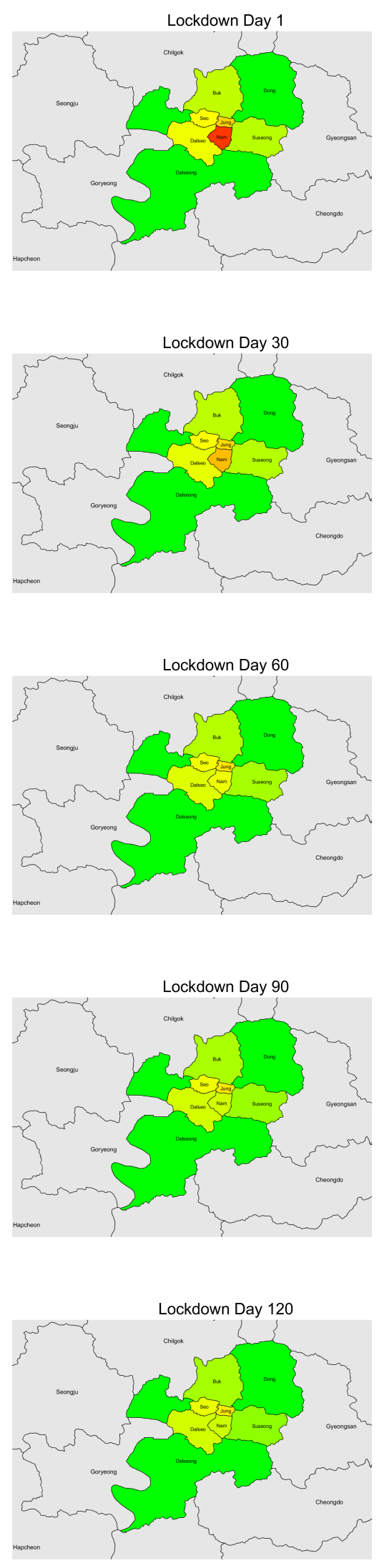

Seoul
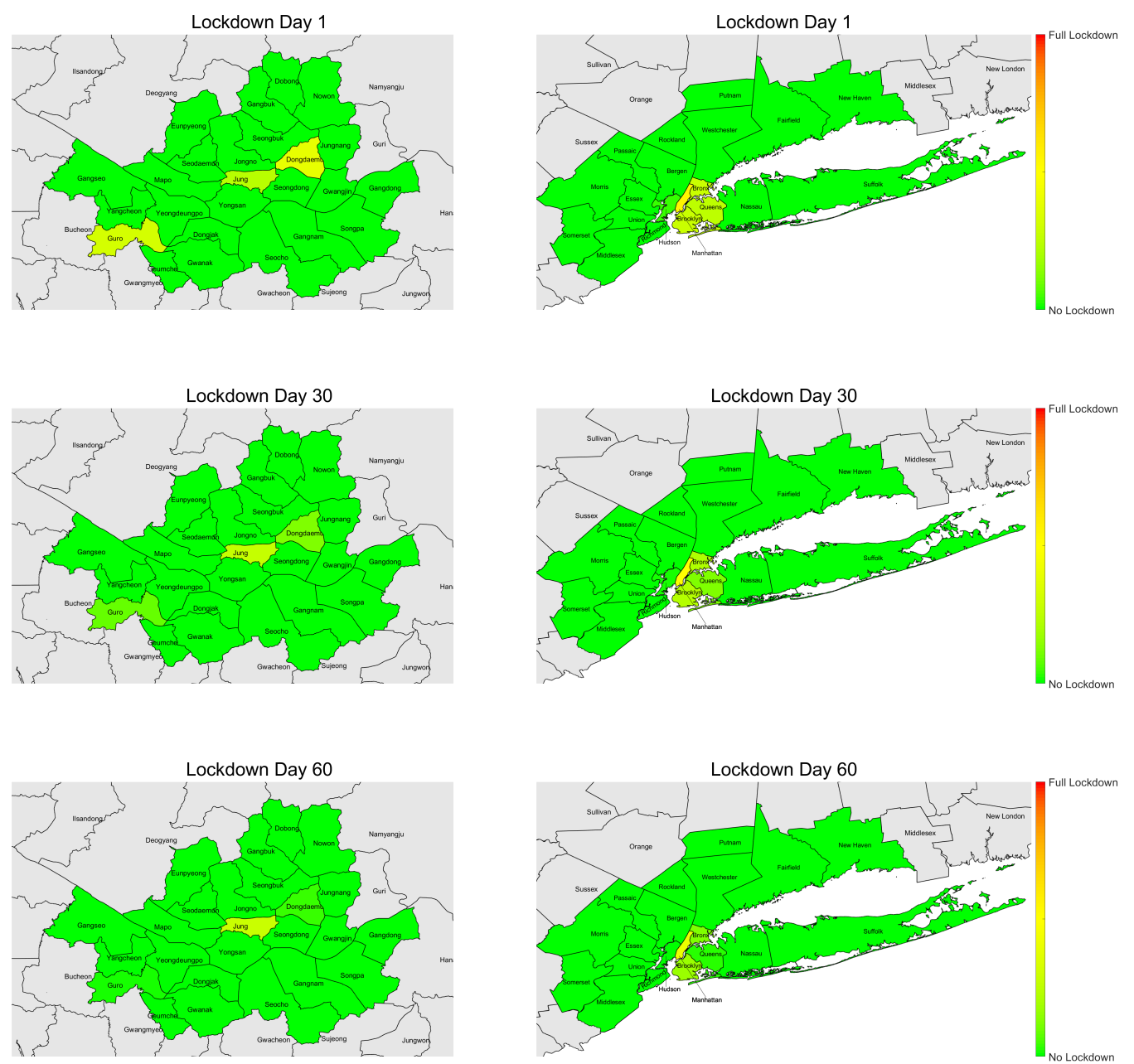

NYC Metro
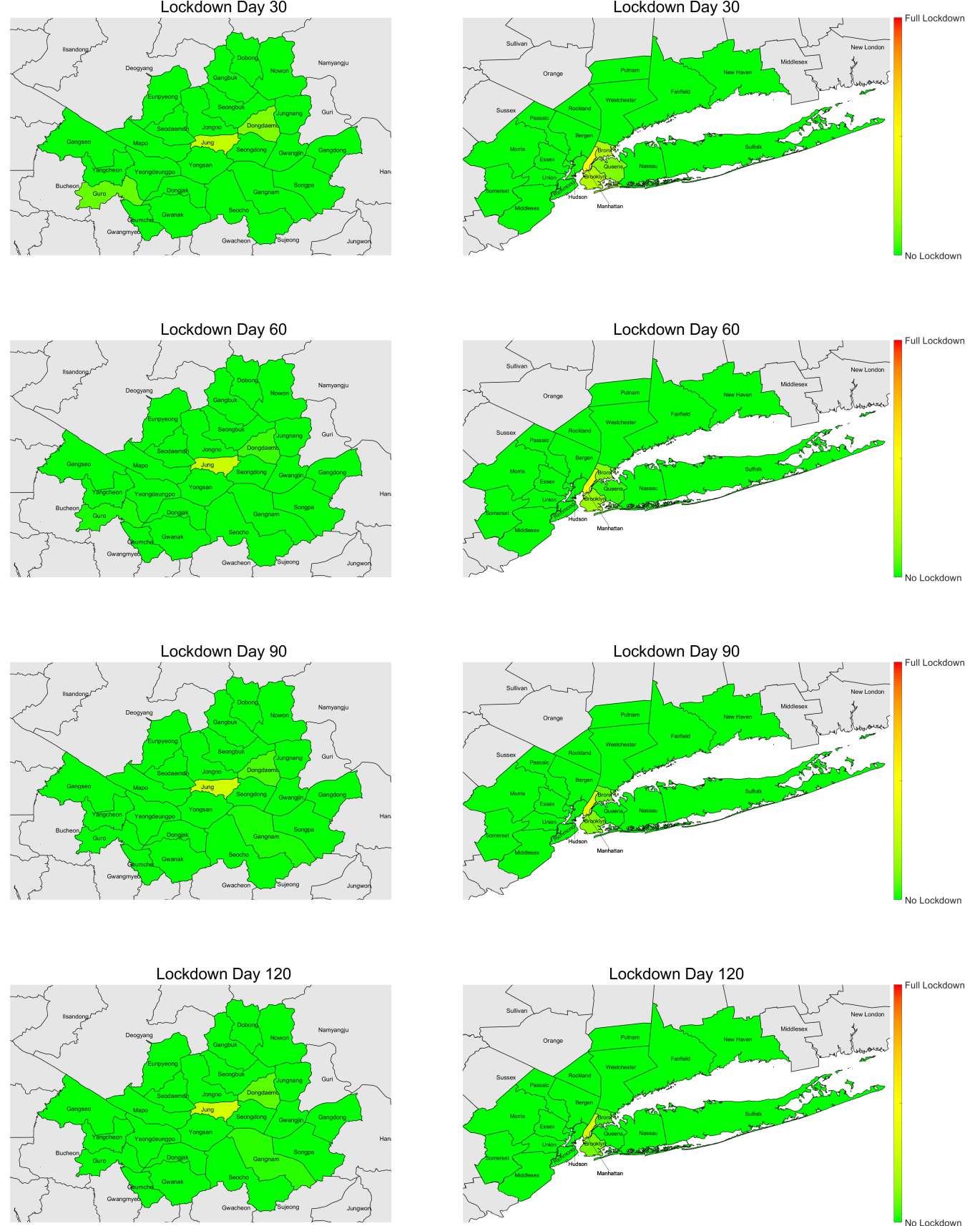

Note: The figure plots the optimal policy in the commuting area at different points in time. Redder colors denote more stringent lockdowns. 
Figure A.4: Commuting and New Daily Cases
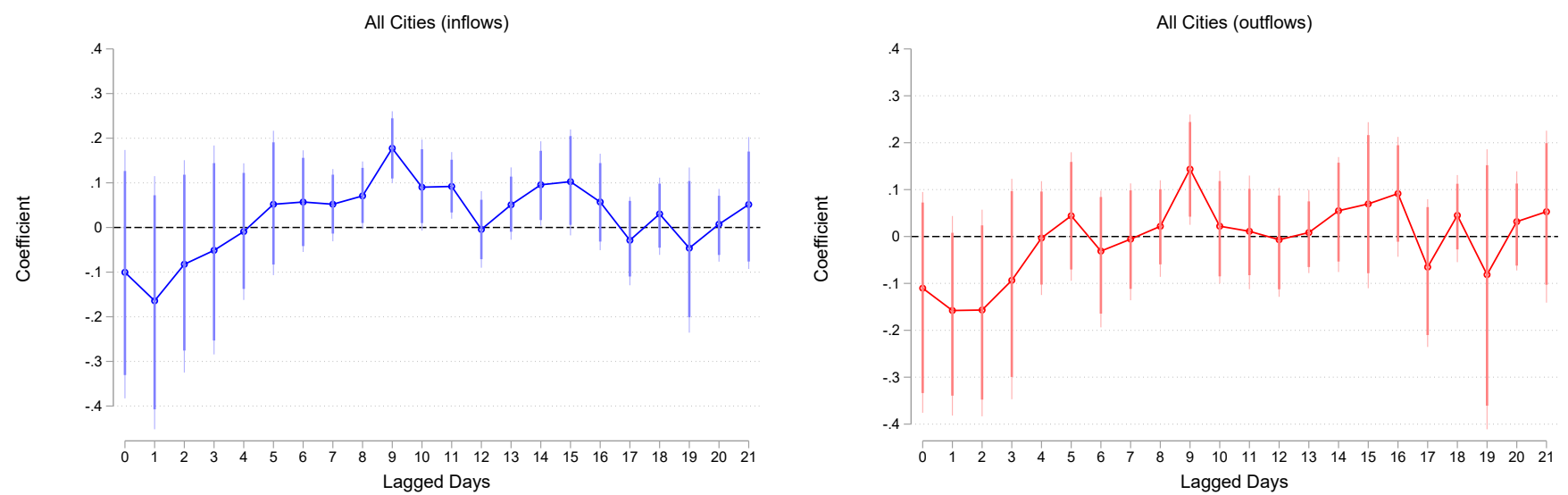

Note: The figure plots the coefficients from (A.15). The left panel reports results using inflows as the independent variable. The right panel reports outflows as the independent variable. The regression pools over the three cities and applies weights so that each city contributes equally. The regression uses data since January 222020 . Error bars show 90 percent (thick) and 95 percent (thin) confidence intervals. Standard errors are clustered by using the block bootstrap to account for a small number of clusters. 
Figure A.5: Robustness

(a) Daegu

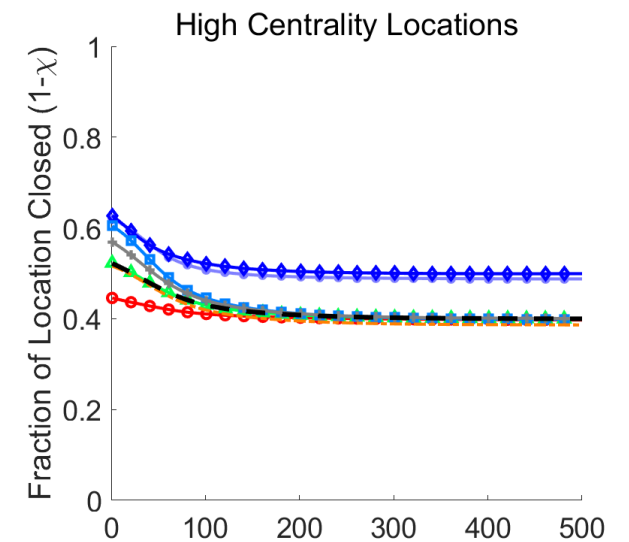

Days since Stay-at-Home Order Began

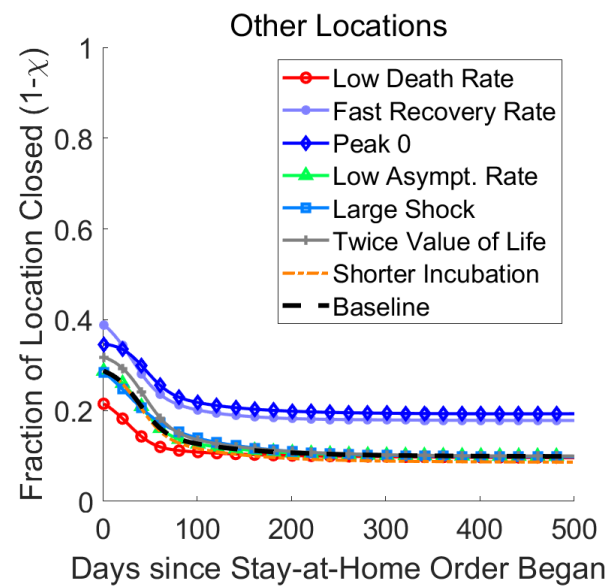

(b) Seoul

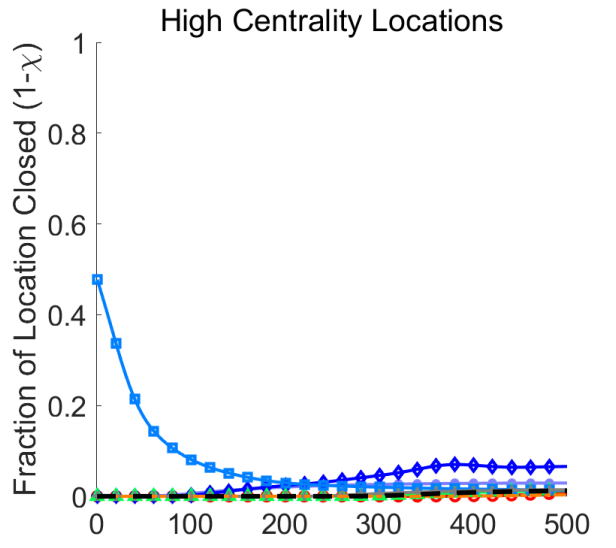

Days since Stay-at-Home Order Began

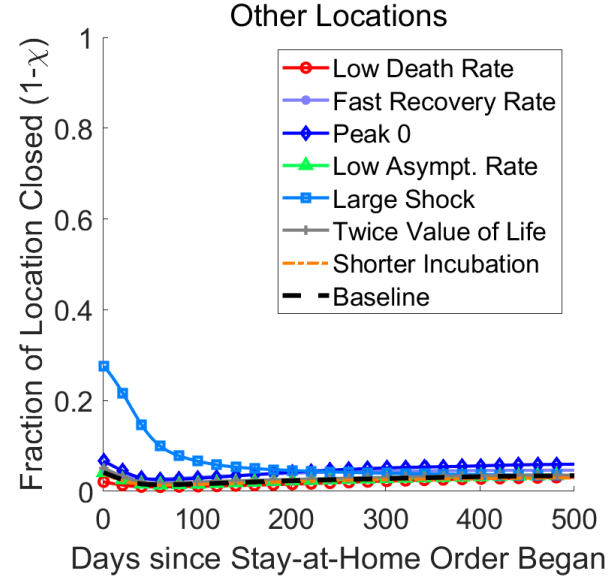

Days since Stay-at-Home Order Began

(c) NYC Metro
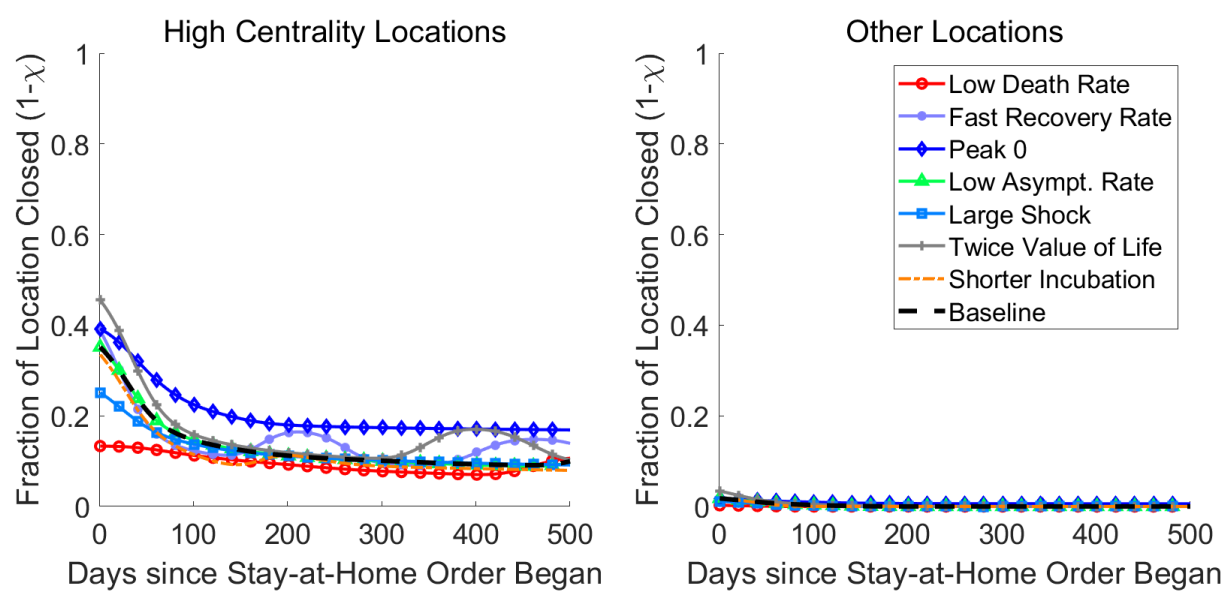

Note: Plotted optimal policies are defined as mean policy for high centrality vs other locations for each city. The different cases correspond to the alternative parametrizations described in Section 3.3 and discussed in Section C. 
Figure A.6: Seoul: Optimal Lockdown in Baseline and Alternative Scenarios

(a) Baseline

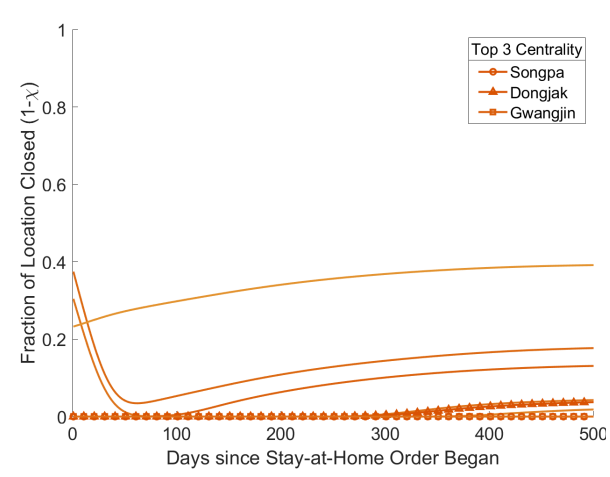

(b) Large Shock

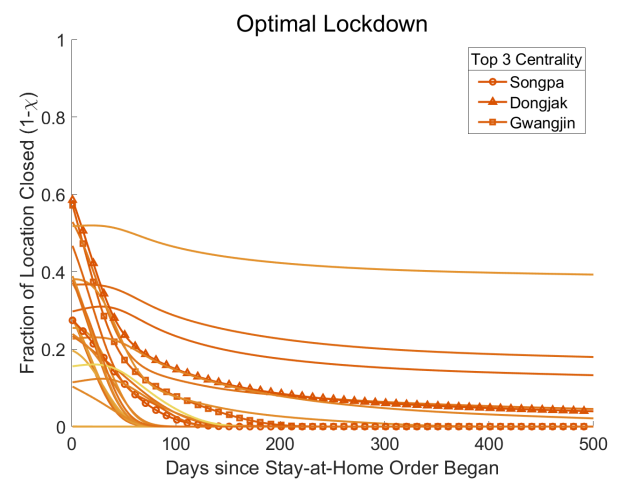

(c) High Value of Life

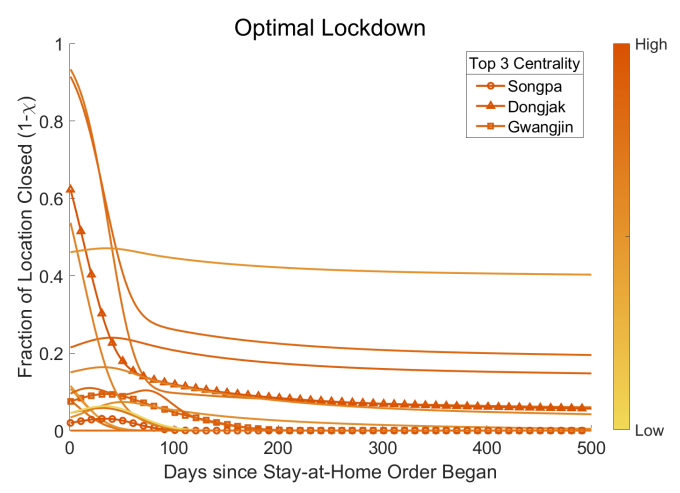

Note: The three panels show the results for Seoul under the baseline calibration (left panel), a large shock infecting $1 \%$ of the population (middle panel) and a value of life that is 100 times the benchmark (right panel). 
Figure A.7: Centrality of Commuting Locations and Optimal Policies (Virus Diffusion through Shopping)

(a) Daegu
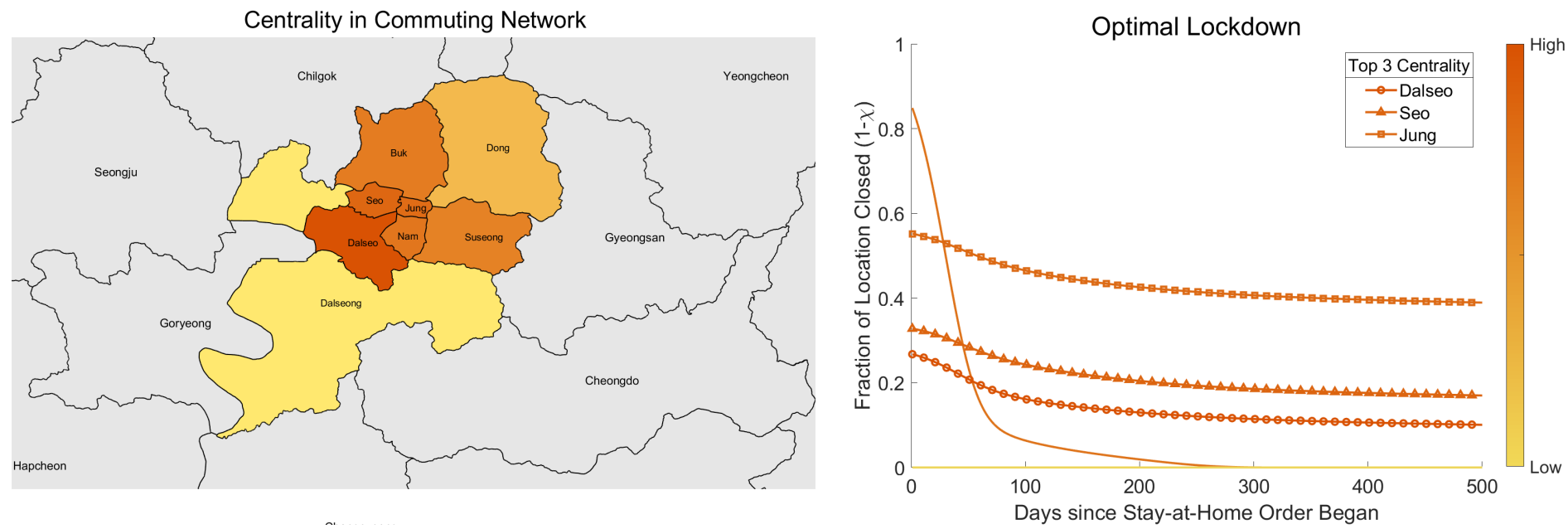

(b) Seoul
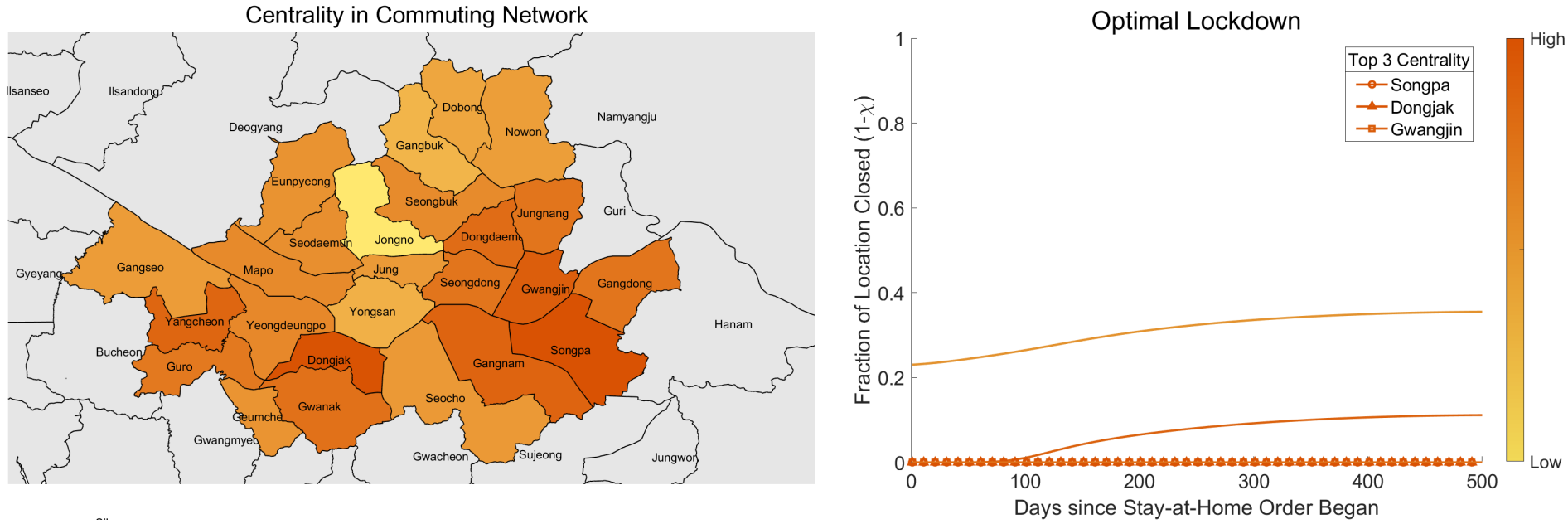

(c) NYC Metro
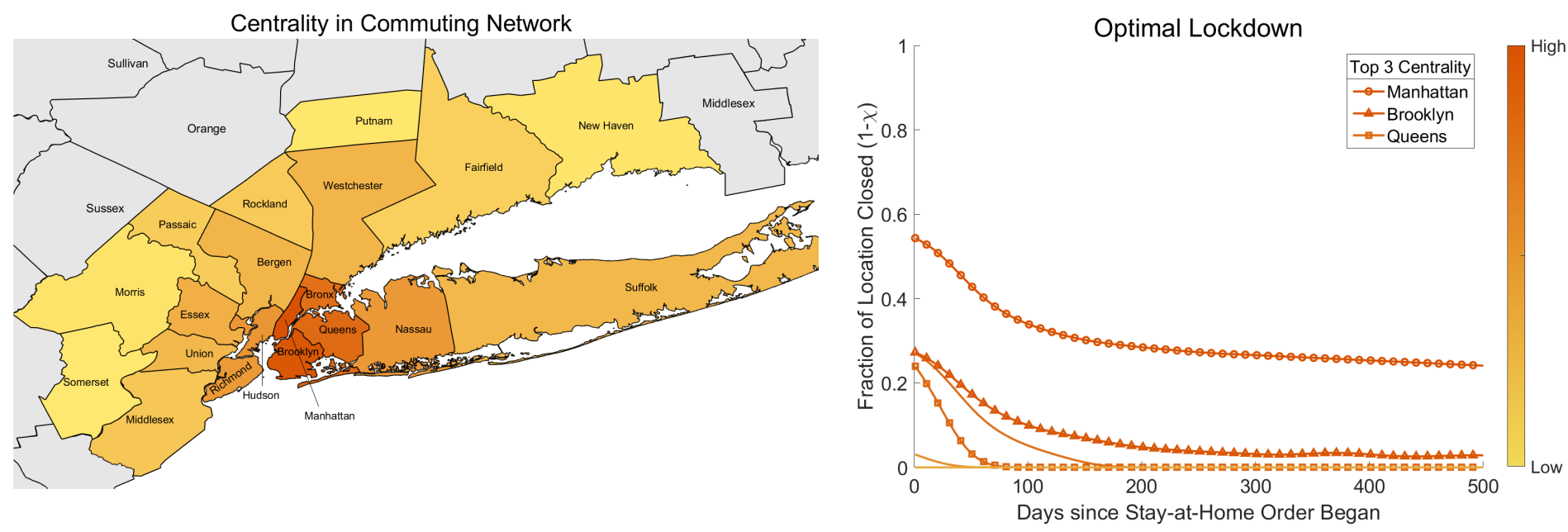

Note: The left panel denotes the (log) centrality of a location (see footnote 18), normalized so that the most central location is 1 . The right panel plots the optimal policies over time for each location in the network. The color of the line represents the centrality of the location in the network. The three most central locations in the network are indicated in the legend. 
Figure A.8: Pareto Frontiers (Virus Diffusion through Shopping)

(a) Daegu

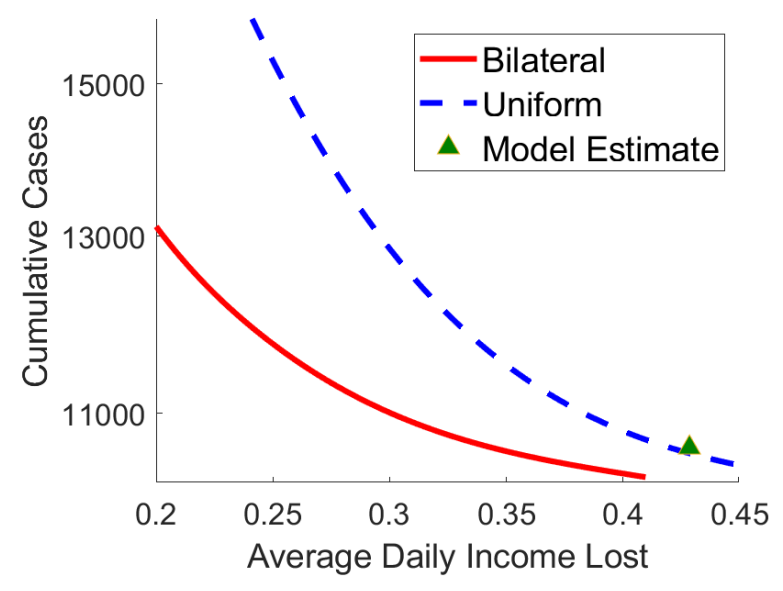

(b) Seoul

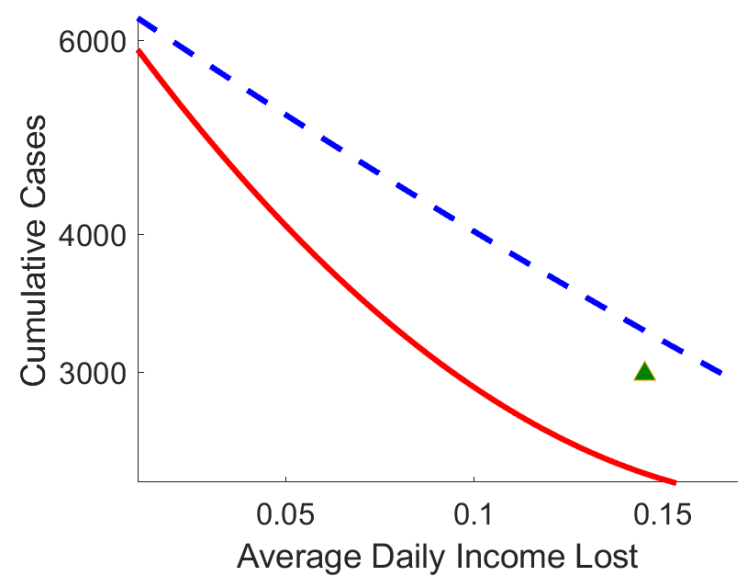

(c) NYC Metro

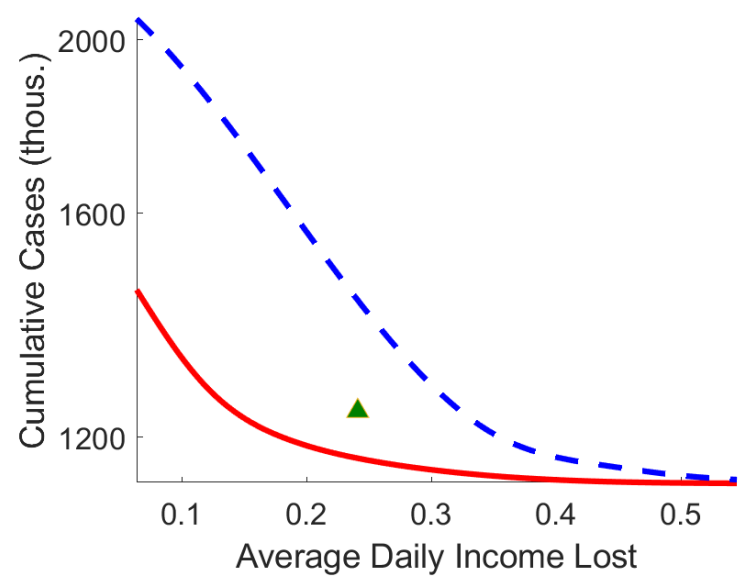

Note: The figures plot the cumulative number of new cases (y-axis, log scale) and the average real income lost per day between the date of the first confirmed case (see Appendix Table A.1) and April 302020 for parametrizations of the value of life $(\omega)$ ranging from 1/100 to 100 times the benchmark, in both the optimal lockdown with space and time variation ("Spatial") and in the spatially uniform optimal solution with time variation only ("Uniform", i.e., the same lockdown across all locations). The green triangle shows the case count and real income lost implied by the estimated model on April 302020. 
Figure A.9: Changes in Commuting Flows: Optimal and Observed (Virus Diffusion through Shopping)

(a) Daegu
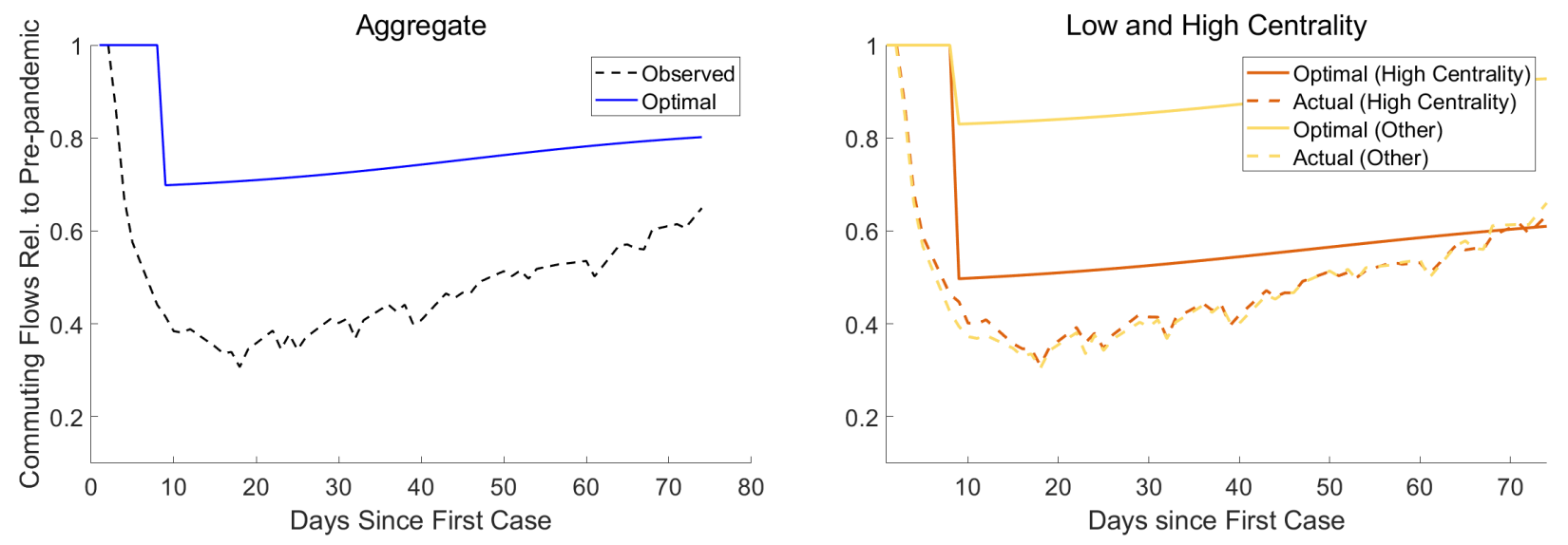

(b) Seoul
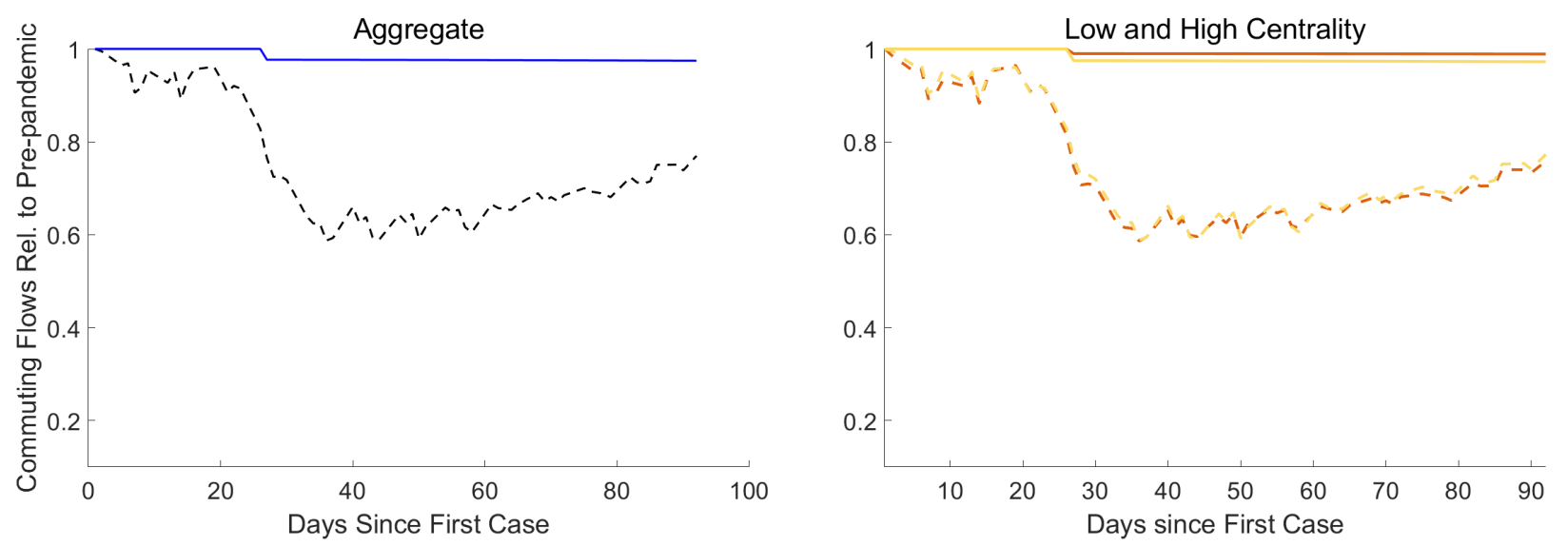

(c) NYC Metro
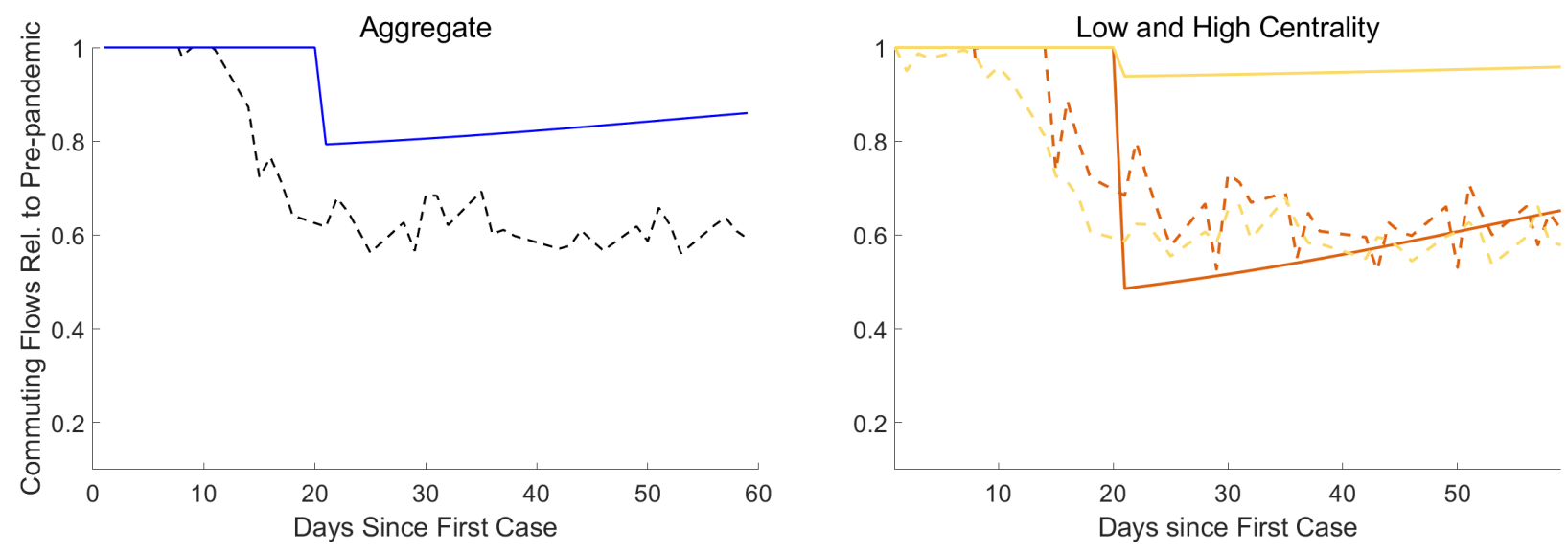

Note: In the left panel, the dashed black line shows the aggregate commuting flows in each city starting from the date of the first confirmed case in each city. The solid and circled blue lines show the aggregate commuting flows implied by the optimal spatial policy. In the right panel, optimal and observed commuting responses are divided by top-3 centrality locations (darker shade) and the other locations. 